\title{
DOCK8 regulates fitness and function of regulatory $T$ cells through modulation of IL-2 signaling
}

\author{
Akhilesh K. Singh, ${ }^{1}$ Ahmet Eken, ${ }^{1}$ David Hagin, ${ }^{1}$ Khushbu Komal, ${ }^{1}$ Gauri Bhise, ${ }^{1}$ Azima Shaji, ${ }^{1}$ \\ Tanvi Arkatkar, ${ }^{1}$ Shaun W. Jackson, ${ }^{1}$ Estelle Bettelli, ${ }^{2}$ Troy R. Torgerson, ${ }^{1,3}$ and Mohamed Oukka, ${ }^{1,3,4}$ \\ 'Seattle Children's Research Institute, Center for Immunity and Immunotherapies, Seattle, Washington, USA. \\ ${ }^{2}$ Benaroya Research Institute, Immunology Program, Seattle, Washington, USA. ${ }^{3}$ Department of Pediatrics and \\ ${ }^{4}$ Department of Immunology, University of Washington, Seattle, Washington, USA.
}

\begin{abstract}
Foxp $3^{+}$Tregs possess potent immunosuppressive activity, which is critical for maintaining immune homeostasis and self-tolerance. Defects in Treg development or function result in inadvertent immune activation and autoimmunity. Despite recent advances in Treg biology, we still do not completely understand the molecular and cellular mechanisms governing the development and suppressive function of these cells. Here, we have demonstrated an essential role of the dedicator of cytokinesis 8 (DOCK8), guanine nucleotide exchange factors required for cytoskeleton rearrangement, cell migration, and immune cell survival in controlling Treg fitness and their function. Treg-specific DOCK8 deletion led to spontaneous multiorgan inflammation in mice due to uncontrolled T cell activation and production of proinflammatory cytokines. In addition, we show that DOCK8-deficient Tregs are defective in competitive fitness and in vivo suppressive function. Furthermore, DOCK8 controls IL-2 signaling, crucial for maintenance and competitive fitness of Tregs, via a STAT5-dependent manner. Our study provides potentially novel insights into the essential function of DOCK8 in Tregs and immune regulation, and it explains the autoimmune manifestations associated with DOCK8 deficiency.
\end{abstract}

Conflict of interest: The authors have declared that no conflict of interest exists.

Submitted: March 29, 2017 Accepted: August 29, 2017 Published: October 5, 2017

Reference information: JCI Insight. 2017;2(19):e94275. https://doi.org/10.1172/jici. insight.94275.

\section{Introduction}

Maintenance of immune homeostasis is essential for keeping the balance between protective immunity and autoimmunity (1-3). Tregs play an indispensable role in immune tolerance, as defective Treg development or function results in inadvertent immune activation and autoimmunity (4-6). Transcription factor Foxp3 has been identified as a master regulator for the development and function of Tregs $(1,2)$. Foxp3 deficiency results in autoimmune disease called immunodysregulation polyendocrinopathy enteropathy X-linked syndrome (IPEX) in humans and Scurfy mice due to lack of functionally competent Tregs (7-9). Naturally occurring Tregs are generated in the thymus, and their development depends on $\mathrm{T}$ cell receptor signaling to the high-affinity self-antigen IL-2 and signaling through the costimulatory receptor CD28 (10).

Tregs constitutively express high-affinity IL-2R. Signaling through IL-2R is critical for the maintenance and function of Tregs in vivo $(11,12)$. More explicitly, IL-2/IL-2R signaling is essential for Treg development in the thymus and for maintaining homeostasis and the competitive fitness of peripheral Tregs. IL-2-mediated signaling contributes to Tregs through activation of transcription factor STAT5, which is essential for Treg development and function (13-15). In conjunction with this, complete deletion of STAT5 resulted in drastic reduction in Tregs (16). Despite recent advances in Treg biology, we still do not fully understand the molecular and cellular mechanisms governing the development and suppressive function of these cells.

DOCK8 interacts with the Rho family small G protein Cdc42 that is required for activation of small G protein from GDP-bound inactive to GTP-bound active form. DOCK8 mutations are associated with autosomal recessive combined immunodeficiency in humans (17-19). DOCK8 deficiency in humans leads to recurrent cutaneous and sinopulmonary infections, atopic dermatitis, elevated serum IgE, and eosinophilia. DOCK8-deficient patients are more prone to cancer, including metastatic squamous carcinoma and B cell lymphoma. The majority of these patients have low numbers of $\mathrm{CD} 4^{+}$and $\mathrm{CD} 8^{+} \mathrm{T}$ cells in the blood. DOCK 8 has been shown to regulate $\mathrm{B}, \mathrm{T}, \mathrm{NKT}$, and NK cells and DCs functions in mice $(17,20-26)$. We have recently uncovered a poten- 
tially novel function of DOCK8 in protective immunity by regulating the generation, survival, and function of ROR $\gamma \mathrm{t}^{+}$innate lymphoid cells (27). Thus, DOCK8 deficiency affects a wide range of immune cells, which hampered our understanding of the precise function of DOCK8 in particular types of immune cells. DOCK8-deficient patients are also more prone to autoimmunity, which could be because of defective Treg function (28). However, defective Treg function in these patients could be due to secondary causes. Therefore, studies that will unequivocally establish the role of DOCK8 in Tregs biology are needed.

To understand the precise role of DOCK8 in Tregs, we generated DOCK8-conditional KO mice and bred them with Foxp3 $3^{\text {Cre }}$ mice to study its specific functions in Tregs. Here, we provide evidence that DOCK8 is essential for the competitive fitness of Tregs and their suppressive functions. Using Foxp $3^{\text {Cre }} \mathrm{D}$ OCK $8^{\mathrm{fl} / \mathrm{fl}}$ mice, we showed that DOCK8 plays a critical role in Tregs through IL-2 signaling in a STAT5-dependent manner. Thus, specific deficiency of DOCK8 in Tregs is associated with multiorgan inflammation due to uncontrolled $\mathrm{T}$ cell activation and production of proinflammatory cytokines.

\section{Results}

DOCK8-deficient mice have reduced Treg frequency and produce less IL-2 upon TCR stimulation. In our mouse colony, we observed that as DOCK8-deficient mice aged, they often develop gastritis (data not shown). Therefore, we hypothesized that DOCK8-deficient animals might have a defect in immune tolerance. Thus, we analyzed Treg frequency in lymphoid organs such as spleen and lymph nodes (LN). As we predicted, DOCK8-deficient animals had a significantly lower frequency of Tregs among CD4 $4^{+} \mathrm{T}$ cells (Figure $1, \mathrm{~A}$ and $\mathrm{B}$ ). To understand the reason behind reduced Treg frequency in DOCK8-deficient animals, we tested whether CD4 ${ }^{+} \mathrm{T}$ cells from these animals could produce normal levels of IL-2 upon T cell receptor (TCR) engagement, as IL-2 is essential for Treg maintenance and function in vivo (11). To our surprise, the frequency of IL- $2^{+} \mathrm{CD} 4^{+} \mathrm{T}$ cells from the DOCK8-deficient group was significantly reduced in comparison with those of WT CD4 ${ }^{+} \mathrm{T}$ cells (Figure 1, C and D). Since, DOCK8-deficient animals have multiple defects and their reduced frequency of $\mathrm{IL}-2^{+} \mathrm{CD} 4^{+} \mathrm{T}$ cells could be due to secondary causes, we decided to revisit this issue by using conditional $\mathrm{KO}$ animals $\left(C D 4^{\mathrm{Cre}} \mathrm{DOCK} 8^{\mathrm{fl} / \mathrm{fl}}\right)$ in which DOCK8 expression is specifically deleted in $\mathrm{T}$ cells. Consistent with $\mathrm{CD}^{+} \mathrm{T}$ cells of DOCK8-deficient mice, $\mathrm{CD} 4^{+} \mathrm{T}$ cells of $\mathrm{CD} 4^{\mathrm{Cre}} \mathrm{DOCK} 8^{\mathrm{fl} / \mathrm{fl}}$ animals also produced significantly less IL-2 than WT CD4 ${ }^{+}$T cells (Figure 1, C and D). These data show that DOCK8 contributes to IL-2 production by $\mathrm{CD} 4^{+} \mathrm{T}$ cells and may indirectly impact the generation and the function of Tregs. These results could explain recent findings that patients with DOCK8 deficiency have reduced numbers of Tregs (28).

Treg-specific deletion of DOCK8 causes T cell activation and autoimmunity. To investigate the function of DOCK 8 in Tregs in vivo, we generated DOCK $8^{\mathrm{f} / \mathrm{fl}}$ mice and crossed them with Foxp3 ${ }^{\mathrm{Cre}}$ mice that express YFP-Cre recombinase fusion protein under the control of the endogenous Foxp3 promoter (29). Both male and female Foxp $3^{\mathrm{Cre}} \mathrm{DOCK} 8^{\mathrm{fl} / \mathrm{fl}}$ mice were born at expected Mendelian ratios and appeared normal at the time of weaning. At 6-8 weeks of age, these mice developed signs of blepharitis and crusting of tail and ears (Figure 1E), and they became moribund at 10-24 weeks after birth (Figure $1 F)$. In the absence of DOCK8 in Tregs, mice also displayed splenomegaly, lymphadenopathy, inflammation in the small intestine and colon, and gastritis (Figure 1G and Supplemental Figure 1A; supplemental material available online with this article; https://doi.org/10.1172/jci.insight.94275DS1), and they had vasculitis in the lungs due to massive infiltration of leukocytes (Figure $1 \mathrm{H}$ ). We also detected significantly increased IgM, IgG, and IgG2c anti-dsDNA autoantibodies in Foxp ${ }^{\mathrm{Cre}} \mathrm{DOCK} 8^{\mathrm{f} / \mathrm{fl}}$ mice relative to age-matched control animals. In addition, we observed elevated anti-Sm/RNP IgM antibodies with a trend toward higher IgG and IgG2c titers (Figure 1I).

Next, we sought to assess whether this severe inflammation was associated with the expansion of $\mathrm{T}$ cells. We observed massive T cell expansion in spleen and lungs with high expression of cell-proliferation marker Ki-67 (Supplemental Figure 1B). The multiorgan inflammation observed in Foxp3 ${ }^{\mathrm{Cre}} \mathrm{DOCK} 8^{\mathrm{f} / \mathrm{ll}}$ mice prompted us to examine whether immune homeostasis was altered in these mice. Foxp3 ${ }^{\mathrm{Cre} D O C K 8^{\mathrm{f} / \mathrm{fl}}}$ mice had more activated CD62 $\mathrm{L}^{\text {low }} \mathrm{CD} 44^{\text {high }}$ effector/memory $\mathrm{T}$ cells and fewer naive $\mathrm{CD} 62 \mathrm{~L}^{\text {high }} \mathrm{CD} 44^{\text {low }}$ cells in comparison with age- and sex-matched control mice (Figure 2, A and B). To determine whether this altered naive/effector switch resulted in proinflammatory cytokine production, we stimulated cells ex vivo and quantified the production of IL-17A and IFN- $\gamma$ by CD4 ${ }^{+} \mathrm{T}$ cells. In line with the activated phenotype, CD ${ }^{+} \mathrm{T}$ cells from Foxp$3^{\mathrm{Cre}} \mathrm{DOCK} 8^{\mathrm{f} / \mathrm{fl}}$ mice produced significantly higher IL-17 and IFN- $\gamma$ (Figure 2, C-E). Thus, DOCK8 deficiency in Tregs leads to systemic and multiorgan inflammation due to uncontrolled activation of $\mathrm{CD}^{+}{ }^{+}$effector $\mathrm{T}$ cells and increased levels of proinflammatory cytokines. 
A

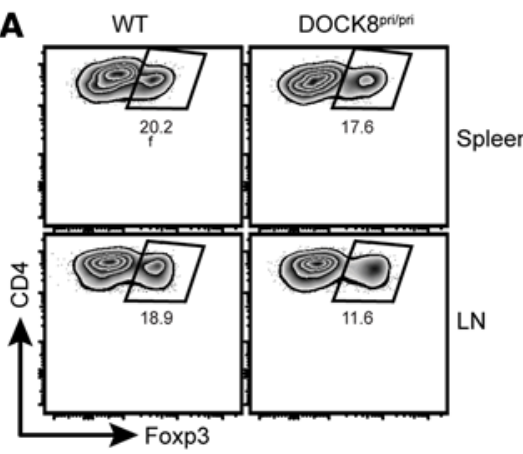

B

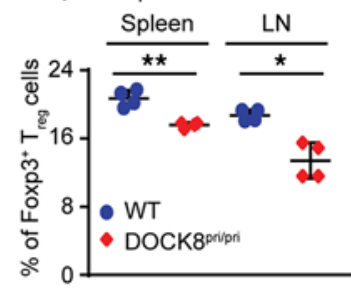

E

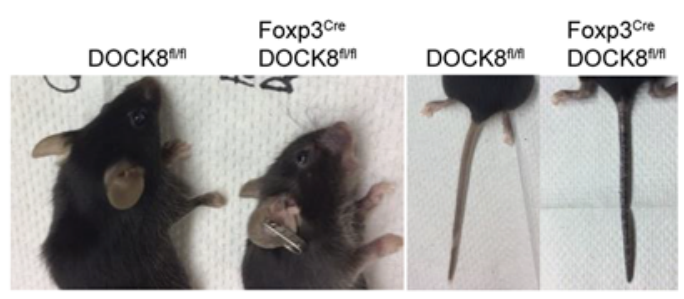

G
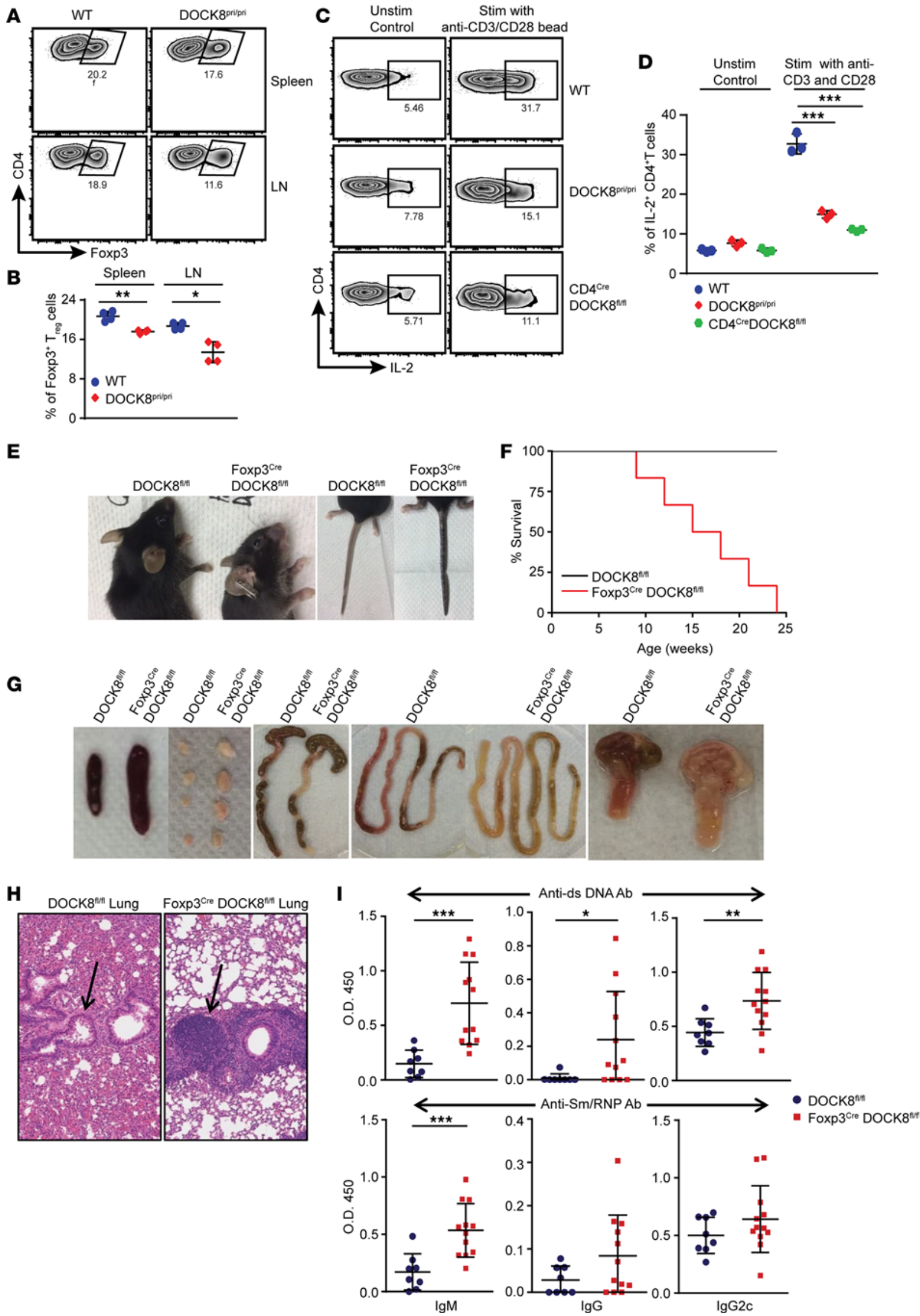
Figure 1. Treg-specific deletion of DOCK8 causes autoimmunity. (A and B) Flow plot and graph showing the frequency of Tregs in the spleen and LNs of WT and DOCK8-deficient mice. Data represents 2 experiments with 4 mice per group. (C and D) DOCK8-deficient T cells have intrinsic defect in IL-2 production in response to TCR stimulation. WT, DOCK $8^{\text {pri/pri, }}$, and CD4 ${ }^{\text {ree DOCK }} 8^{\text {fl/fl }}$ animal LN cells were stimulated with anti-CD3 and anti-CD28 for 24 hours, followed by 4-hour stimulation in the presence of PMA and lonomycine and then intracellular cytokine staining for IL-2. Data represents 3 experiments with 3 mice per group. (E) Representative image of small mouse size, crusting of tail, and skin lesions that develop around ears of 8-week-old Foxp3 $3^{\text {CreDOCK }} 8^{\text {fl/ }}$ ${ }^{\mathrm{fl}}$ mice compared with control (DOCK8 ${ }^{\mathrm{fl} / \mathrm{fl})}$ mice. (F) Percent survival and (G) Representative images showing splenomegaly (first from left), lymphadenopathy (second from left), and inflammation in small intestine (third and fourth from left) and stomach (fifth from left) in 8-week-old Foxp $3^{\text {Cre }}$ DOCK ${ }^{\text {fl/fl }}$ mice compared with control mice. (H) Representative section of lung stained with H\&E depicting higher lymphocytic infiltration (pointed by arrow) in 8-week-

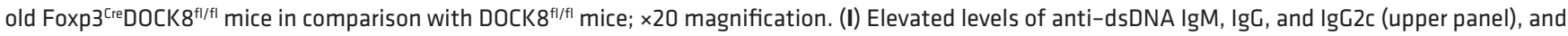
anti-Sm/RNP IgM, with a trend toward increased anti-Sm/RNP IgG and IgG2c Ab (lower panel) in Foxp3 ${ }^{\text {CreDOCK }}{ }^{\mathrm{fl} / \mathrm{fl}}$ mice compared with age-matched controls. Data represent 5-12 mice per group. These data shown are the mean \pm SD. Statistics were performed with Prism software by using $t$ test (B and $\mathbf{I}$ ) and 1-way ANOVA (D). ${ }^{*} P<0.05,{ }^{* *} P<0.01,{ }^{* * *} P<0.001$.

DOCK8 deficiency does not impact the development of Tregs. Treg development, homeostasis, and function are dependent on the Treg-specific transcription factor Foxp3. Therefore, loss of Foxp3 expression in Tregs or a defect in its function leads to autoimmunity in a T cell-dependent fashion (10). Consequently, we sought to determine whether Treg development in the thymus was altered in Foxp ${ }^{\text {Cre }}$ Dock $8^{\mathrm{f} / \mathrm{fl}}$ mice. We did not find any significant difference in the number of Tregs generated in the thymus between Foxp $3^{\text {Cre }}$ Dock $8^{\mathrm{f} / \mathrm{fl}}$ mice and control mice. Consistent with the thymus, expression of Foxp3 by DOCK8-deficient Tregs in blood, spleen, LNs, and lungs was not altered in comparison with WT Tregs (Figure 3, A and B, and Supplemental Figure 2A). These findings imply that DOCK8-deficient Tregs are generated normally in the thymus and are also able to egress to peripheral organs.

We next compared the expression of a number of cell surface markers on Tregs from Foxp $3^{\text {Cre }} \mathrm{D}$ $\mathrm{OCK} 8^{\mathrm{f} / \mathrm{fl}}$ and WT mice. To our surprise, $\mathrm{YFP}^{+}$Tregs from Foxp $3^{\mathrm{Cre} D O C K} 8^{\mathrm{fl} / \mathrm{fl}}$ mice expressed significantly less CD25 (IL-2R $\alpha$ ) than their WT counterparts (Figure 3C). It is well established that CD25 expression is essential for IL-2-mediated signaling, which is crucial for Treg homeostasis and competitive fitness in vivo (11). Tregs from IL-2- or IL-2R $\alpha$-deficient mice acquire an activated phenotype with decreased expression of CD62L and enhanced expression of CTLA-4, CD69, and CD103 (11). Interestingly, DOCK8-deficient Tregs also display an activated phenotype with increased expression of CTLA-4, ICOS, CD69, and KLRG1 and less expression of CD62L (Figure 3C). These results suggested that DOCK8 was either controlling the CD25 expression directly at transcription level or indirectly through IL-2-mediated signaling. However, CD25 transcript levels were comparable between WT and DOCK8-deficient Tregs (Supplemental Figure 2B). Thus, it is likely that DOCK8 controls CD25 expression levels via a post-transcriptional mechanism, probably through IL-2-mediated signaling.

DOCK8 is dispensable for Treg homing. Since, DOCK8 has been shown to regulate interstitial DC migration (21), it raised the question whether DOCK8-deficient Tregs could home normally to lymphoid and nonlymphoid tissues under steady state. Although, our aforementioned results (Figure 3) have indicated that Tregs of Foxp3 $3^{\text {Cre }}$ Dock $8^{\mathrm{fl} / \mathrm{fl}}$ mice were present in normal numbers in lymphoid and nonlymphoid organs, it is possible that the ongoing inflammation and the increased number of activated $\mathrm{T}$ cells may have changed the trafficking behavior of these Treg. Thus, we generated Foxp3 $3^{\text {CreERT- }}$ ${ }^{2}$ DOCK $8^{\mathrm{f} / \mathrm{fl}} \mathrm{R} 26 \mathrm{RFP}$ mice by breeding Foxp3 ${ }^{\mathrm{CreERT} 2} \mathrm{DOCK} 8^{\mathrm{f} / \mathrm{fl}}$ mice with ROSA 26 RFP reporter mice. These mice were born normally, and we did not observe any sign of inflammation. We evaluated the deletion of DOCK8 in Foxp $3^{\text {CreERT2}}$ DOCK $8^{\mathrm{fl} / \mathrm{fl}}$ R26RFP mice by the appearance of RFP after tamoxifen injections (Figure 4A). We also confirmed whether $\mathrm{CD}^{+} \mathrm{RFP}^{+}$cells were indeed Tregs by perform-

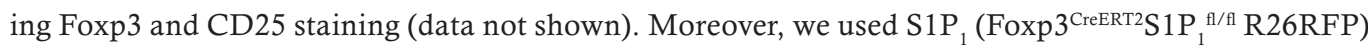
as a positive control where it is required for the egress of cells from LN to peripheral organs (ref. 30 and our unpublished data [AE, AKS, and MO]). Thus, we injected tamoxifen in control, Foxp3 ${ }^{\text {CreERT- }}$ ${ }^{2} \mathrm{DOCK} 8^{\mathrm{fl} / \mathrm{fl}} \mathrm{R} 26 \mathrm{RFP}$, and Foxp3 ${ }^{\mathrm{CreERT}} \mathrm{S}_{1} \mathrm{P}_{1}^{\mathrm{fl} / \mathrm{fl}} \mathrm{R} 26 \mathrm{RFP}$ mice and analyzed spleens, LNs, and lungs at day 10 after injection. Consistent with data in Figure 3, acute deletion of DOCK 8 did not change the frequency of Tregs in indicated organs. However, as expected, acute deletion of $\mathrm{S}_{1} \mathrm{P}_{1}$ resulted in the accumulation of Tregs in LN and a substantial decrease of Tregs in spleens and lungs (Figure 4B).

We also analyzed Tregs from lungs of Foxp $3^{\mathrm{CreERT} 2} \mathrm{Cdc} 42^{\mathrm{fl} / \mathrm{fl}}$ mice and Foxp $3^{\mathrm{CreERT} 2} \mathrm{DOCK} 8^{\mathrm{f} / \mathrm{fl}}$ mice beyond day 10 after tamoxifen infusion. We tested the function of Cdc42 in Tregs because it had been previously shown that $\mathrm{T}$ cell-specific deletion of Cdc42 resulted in decreased $\mathrm{T}$ cell trafficking and cell survival (31). As expected, the number of Tregs was drastically decreased in lungs of Foxp $3^{\mathrm{CreERT}} \mathrm{Cdc} 42^{\mathrm{f} / \mathrm{fl}}$ mice 
A
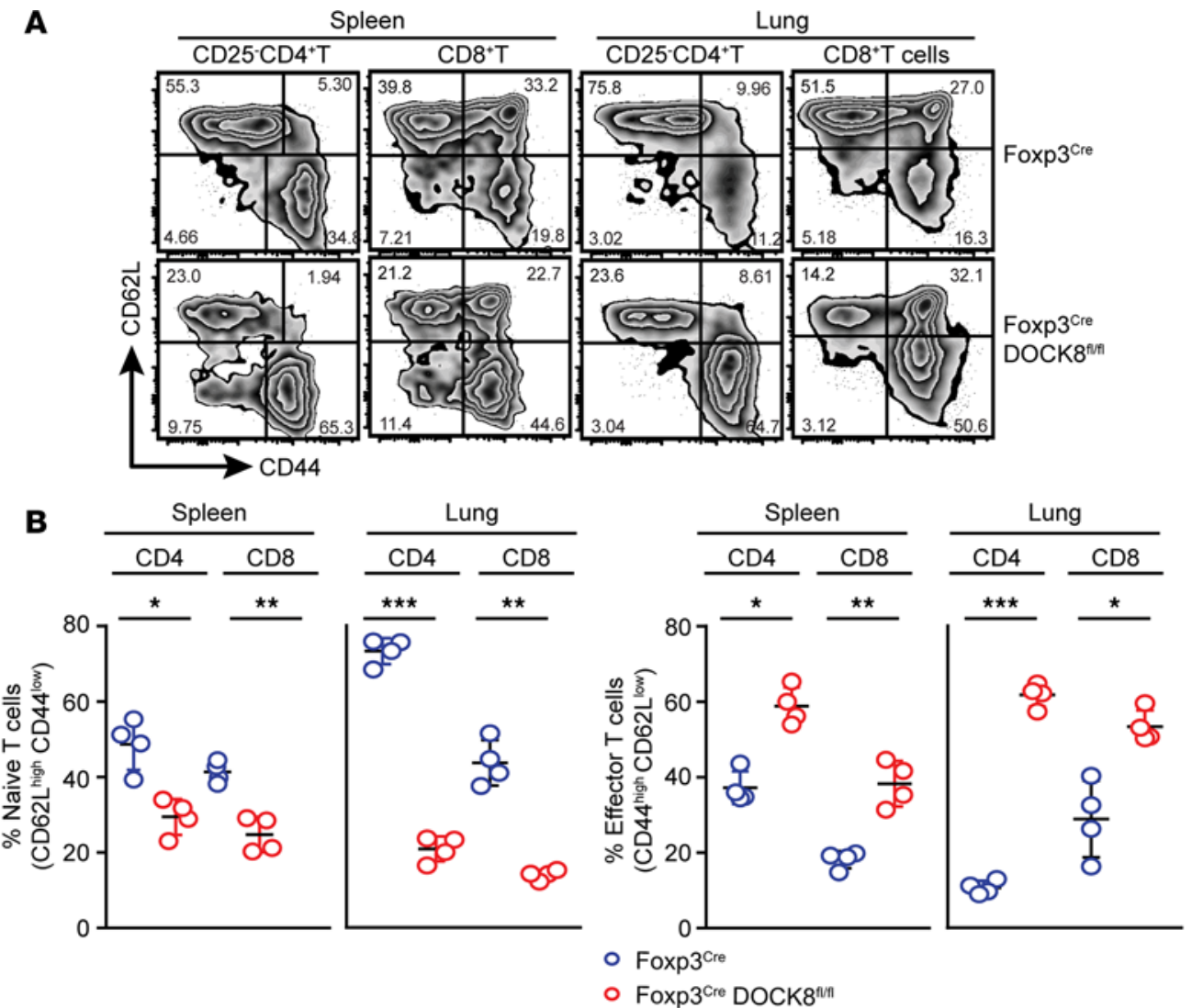

- Foxp3 $3^{\mathrm{Cre}} \mathrm{DOCK} 8^{\text {nn }}$

C

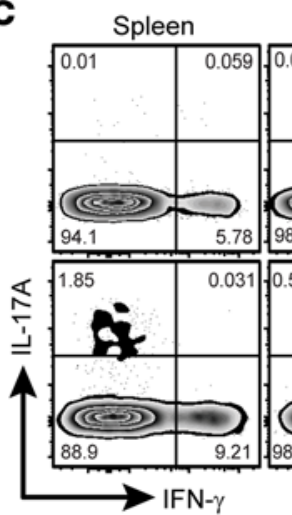

D
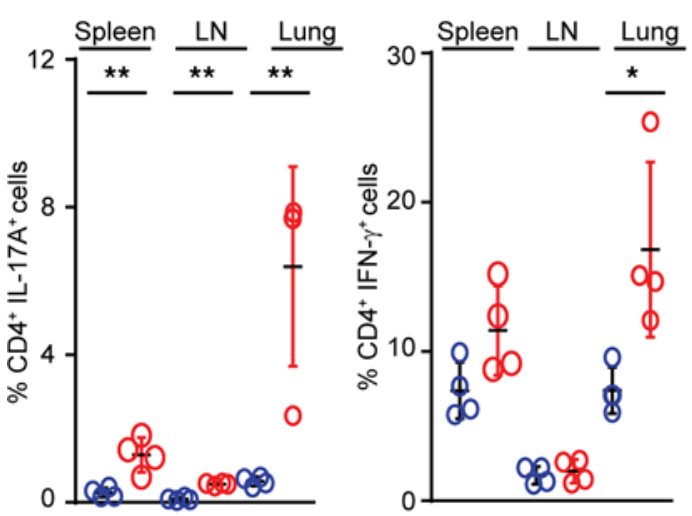

E
Figure 2. DOCK8-deficient Tregs failed to control $T$ cell activation. (A and $\mathbf{B}$ ) $\mathrm{T}$ cells are activated in Foxp $3^{\text {Cre }} \mathrm{D}$ OCK $8^{f / / f l}$ mice. Spleen and lung samples from control and Foxp $3^{\mathrm{Cre}} \mathrm{DOCK} 8^{\mathrm{fl} / \mathrm{fl}}$ mice were analyzed for the expression of CD44 and CD62L on CD25-CD4 $4^{+}$or $\mathrm{CD}^{+} \mathrm{T}$ cells. (A) FACS plot and (B) quantification of the frequency of naive (CD44 ${ }^{\text {low }}$ CD62 $L^{\text {high }}$ ) versus effector/memory (CD44 ${ }^{\text {high }} \mathrm{CD62L}^{\text {low }}$ ) cells in the spleen and lung of control and Foxp3 $3^{\mathrm{Cre}} \mathrm{DOCK} 8^{\mathrm{fl} / \mathrm{fl}}$ mice. Data represents at least 3 independent experiments with 4 mice per group. (C-E) Spleen, LN, and lung cells were stimulated with $50 \mathrm{ng} /$ $\mathrm{ml} \mathrm{PMA}$ and $1 \mu \mathrm{g} / \mathrm{ml}$ lonomycine in the presence of Golgi stop for 4 hours at $37^{\circ} \mathrm{C}$ in $\mathrm{CO}_{2}$ incubator; then, IL-17 and IFN- $\gamma$ expressions in $\mathrm{CD}^{+} \mathrm{T}$ cells were analyzed by flow cytometry. (C) FACS plot, (D) frequency, and (E) absolute number of IL-17+ and/or IFN$\gamma^{+} \mathrm{CD} 4^{+} \mathrm{T}$ cells in the spleen, $\mathrm{LN}$, and lung of control and Foxp3 $3^{\mathrm{Cre}} \mathrm{DOCK} 8^{\mathrm{fl} / \mathrm{fl}}$ mice. Data represent at least 4 independent experiments with minimum of 3 mice per group. The data shown are the mean \pm SD. Statistics were performed with Prism software by using $t$ test. ${ }^{*} P<0.05$, ${ }^{* *} P<0.01$, ${ }^{* * *} P<0.001$. 
treated with tamoxifen, whereas the number of Tregs of Foxp $3^{\mathrm{CreERT} 2} \mathrm{DOCK} 8^{\mathrm{fl} / \mathrm{fl}}$ mice was comparable with WT mice. However, we observed a significant increase of total Tregs in the lungs of Foxp $3^{\text {CreERT2}}$ DOCK $8^{\mathrm{f} / \mathrm{fl}}$ mice due to an increase of effector T cell infiltration (Figure 4, C and D). Altogether, our data suggest that DOCK8 is dispensable for the homing or trafficking of Tregs.

Tregs require DOCK8 for optimal STAT5 activation and fitness. As IL-2-mediated signaling via STAT5 activation is essential for Treg development and homeostasis, we tested whether DOCK8 was involved in IL-2mediated signaling. Thus, we stimulated freshly isolated CD4 ${ }^{+} \mathrm{T}$ cells with IL-2 and assessed STAT5 phosphorylation (pY $\mathrm{P}^{694}$-STAT5) by flow cytometry (Figure 5, A-C). Consistent with previous literature, a very low dose of IL-2 induced STAT5 phosphorylation in WT Tregs; however, IL-2-mediated STAT5 activation was severely impaired in DOCK8-deficient Tregs (Figure 5, A-C). Moreover, similar to DOCK8-defficient Tregs, the knockdown of DOCK8 expression in the $\mathrm{T}$ cell line also resulted in impaired IL-2-mediated STAT5 activation (Supplemental Figure 3). These data indicate that DOCK8 is required for optimal STAT5 activation upon IL-2 stimulation.

Our observation that Tregs from Foxp $3^{\mathrm{Cre}} \mathrm{DOCK} 8^{\mathrm{fl} / \mathrm{fl}}$ mice exhibit decreased IL-2-mediated STAT5 phosphorylation and an activated phenotype suggests that DOCK8 may enhance IL-2 signaling, which is required for the competitive fitness, survival, and expansion of Tregs. Therefore, we expect that, under a competitive setting and noninflammatory conditions, DOCK8-deficient Tregs will be outcompeted by WT Tregs. To address this possibility, we utilized Foxp3 ${ }^{\text {Cre/WT }}$ DOCK $8^{\mathrm{f} / \mathrm{fl}}$ heterozygous female mice, in which both DOCK8-sufficient and DOCK8-deficient Tregs coexist due to random inactivation of the X chromosome. As expected, Foxp $3^{\mathrm{Cre} / \mathrm{WT}} \mathrm{DOCK} 8^{\mathrm{fl} / \mathrm{fl}}$ female mice were healthy and did not show any signs of lymphoproliferative disease. Analysis of $\mathrm{CD} 45^{+} \mathrm{CD} 4^{+} \mathrm{Foxp} 3^{+}$Tregs from thymus, spleens, and LNs of 8-weekold heterozygous female mice showed that the DOCK8-deficient $\left(\mathrm{CD} 45^{+} \mathrm{CD} 4^{+} \mathrm{Foxp}^{+}\right.$and $\left.\mathrm{YFP}^{+}\right) \mathrm{Treg}$ frequency and absolute numbers were significantly lower than those of WT Tregs $\left(\mathrm{CD} 45^{+} \mathrm{CD} 4^{+} \mathrm{Foxp} 3^{+}\right.$ and $\mathrm{YFP}^{-}$) (Figure 5, D, E, and G). Interestingly, in Foxp3 ${ }^{\mathrm{Cre} / \mathrm{WT}} \mathrm{DOCK} 8^{\mathrm{f} / \mathrm{fl}}$ heterozygous female mice, DOCK8-deficient Tregs have lower expressions of Foxp3 and CD25 in comparison with DOCK8-sufficient Tregs (Figure 5, D, F, and H). Thus, these findings suggest that DOCK8 function in Tregs regulate their survival and expansion by enhancing IL-2-mediated signaling.

Likewise, IL-2-driven STAT5 phosphorylation was significantly impaired in DOCK8-deficient Tregs in comparison with DOCK8-sufficient Tregs (Figure 5, I and J). Next, we attempted to rescue IL-2 signaling defects by infusing IL-2 and anti-IL-2 mAb (JES6-1A12) complexes to expand Tregs in vivo. However, in this setting, although DOCK8-deficient Tregs expanded in response to IL-2/IL-2 antibody complexes, this expansion was drastically reduced in comparison with the in vivo expansion of DOCK8-sufficient Tregs (Figure $5 \mathrm{~K}$ ). We also compared in vitro proliferative potential of DOCK8-deficient Tregs by stimulating them with anti-CD3/CD28-coated beads along with IL-2. Consistent with defective in vivo proliferation, DOCK8-deficient Tregs did not proliferate in vitro as well as DOCK8-sufficient Tregs (Figure 5L). Furthermore, we found that IL-2 and IL-2 mAb complex injections failed to rescue Treg defects upon acute deletion of DOCK8 (Supplemental Figure 4, A-D).

Immunosuppressive functions are impaired in Tregs lacking DOCK8. We investigated what functions of Tregs were affected by DOCK8 deficiency. We first investigated the in vitro immune-suppressive activity of DOCK8-deficient Tregs. Thus, we measured the proliferation of in vitro-activated WT CD4 ${ }^{+} \mathrm{T}$ cells in the presence of either WT or DOCK8-deficient Tregs. The proliferation of WT CD4 ${ }^{+} \mathrm{T}$ cells was analyzed by flow cytometry based on the dilution of cell tracer fluorescence intensity. The percentage of dividing effector cells in culture with DOCK8-deficient Tregs was comparable with effector cells cocultured with DOCK8-sufficient Tregs (Figure 6A). Thus, it appears that, at least in vitro, DOCK 8 is not required for Treg-suppressive activity.

Aforementioned results prompted us to examine Treg immunoregulatory function using an in vivo model because conditional deletion of DOCK8 in Tregs renders mice susceptible to inflammation, despite having normal Treg numbers and frequency. Therefore, we used experimental autoimmune encephalomyelitis (EAE) models to address this question because, in this model, effector $\mathrm{CD} 4^{+} \mathrm{T}$ cells first migrate to the $\mathrm{CNS}$ and initiate disease, followed by migration of Tregs, which is critical for limiting the inflammatory response in the CNS and initiating the recovery phase of the disease (32). We directly addressed this by inducing EAE via myelin oligodendrocyte $\left(\mathrm{MOG}_{35-55}\right)$ peptide and complete Freund's adjuvant (CFA) emulsion immunized in mice after acute deletion of DOCK 8 with 4 consecutive tamoxifen injections (Figure $6 \mathrm{~B}$ ). Foxp3 ${ }^{\text {CreERT2}}$ DOCK8 $8^{\mathrm{f} / \mathrm{fl}}$ mice developed significantly more severe EAE in comparison with control tamoxifen-injected Foxp3 ${ }^{\text {CreERT2 }}$ mice (Figure 6C). This observation could be due to reduced numbers of DOCK8-deficient Tregs in CNS of Foxp3 $3^{\text {CreERT }}$ 
A
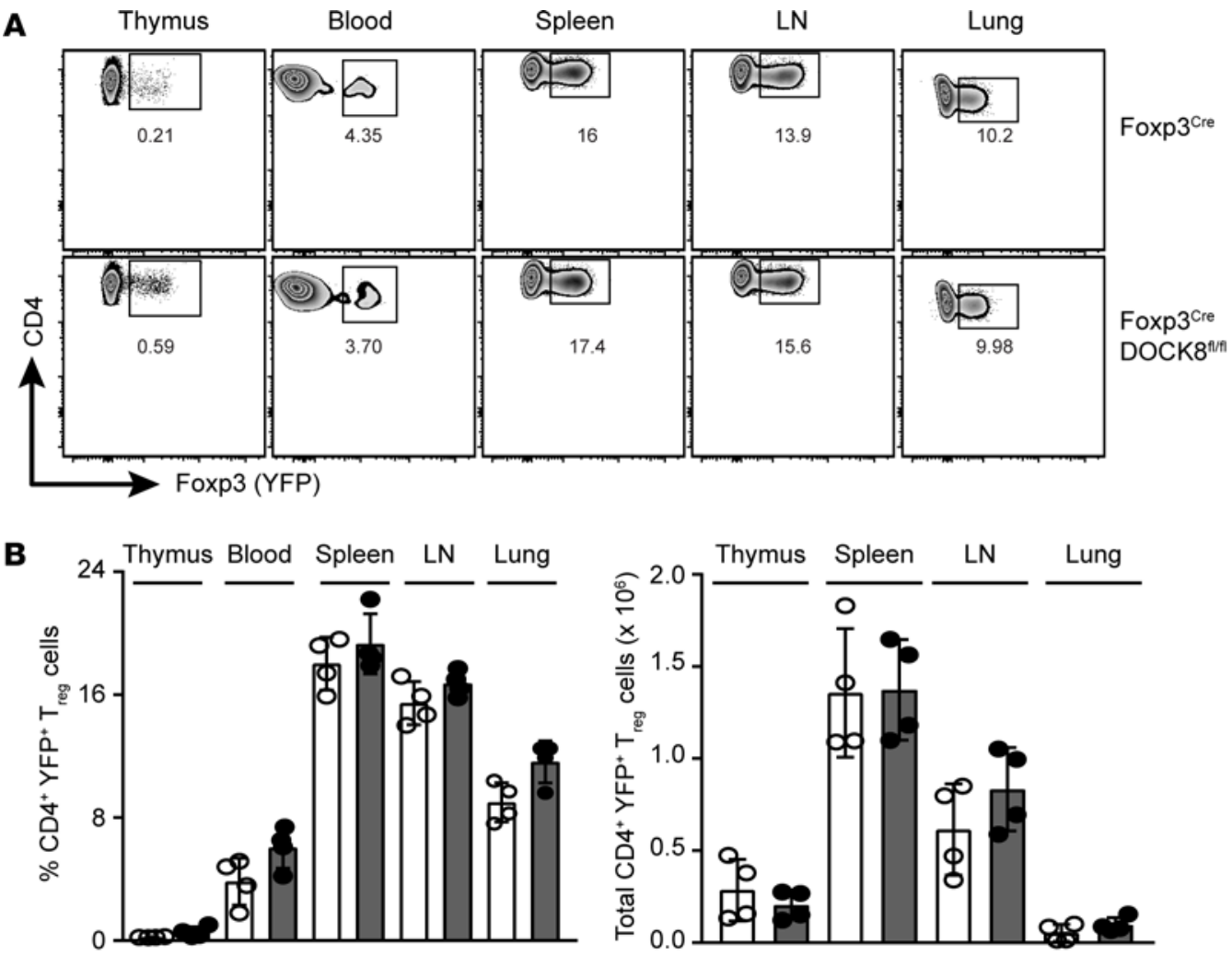

C
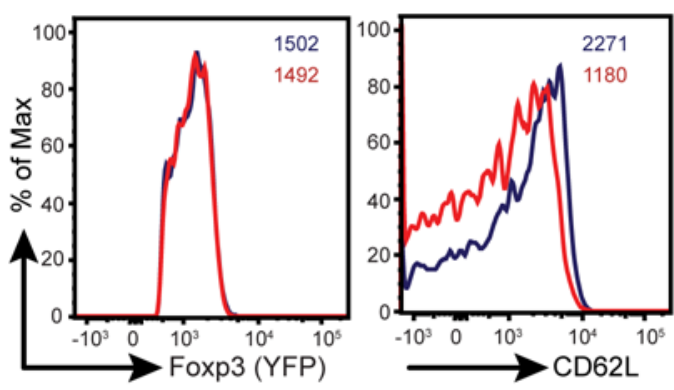

Foxp3 $3^{\text {Cre }}$ DOCK8 $8^{\text {firf }}$
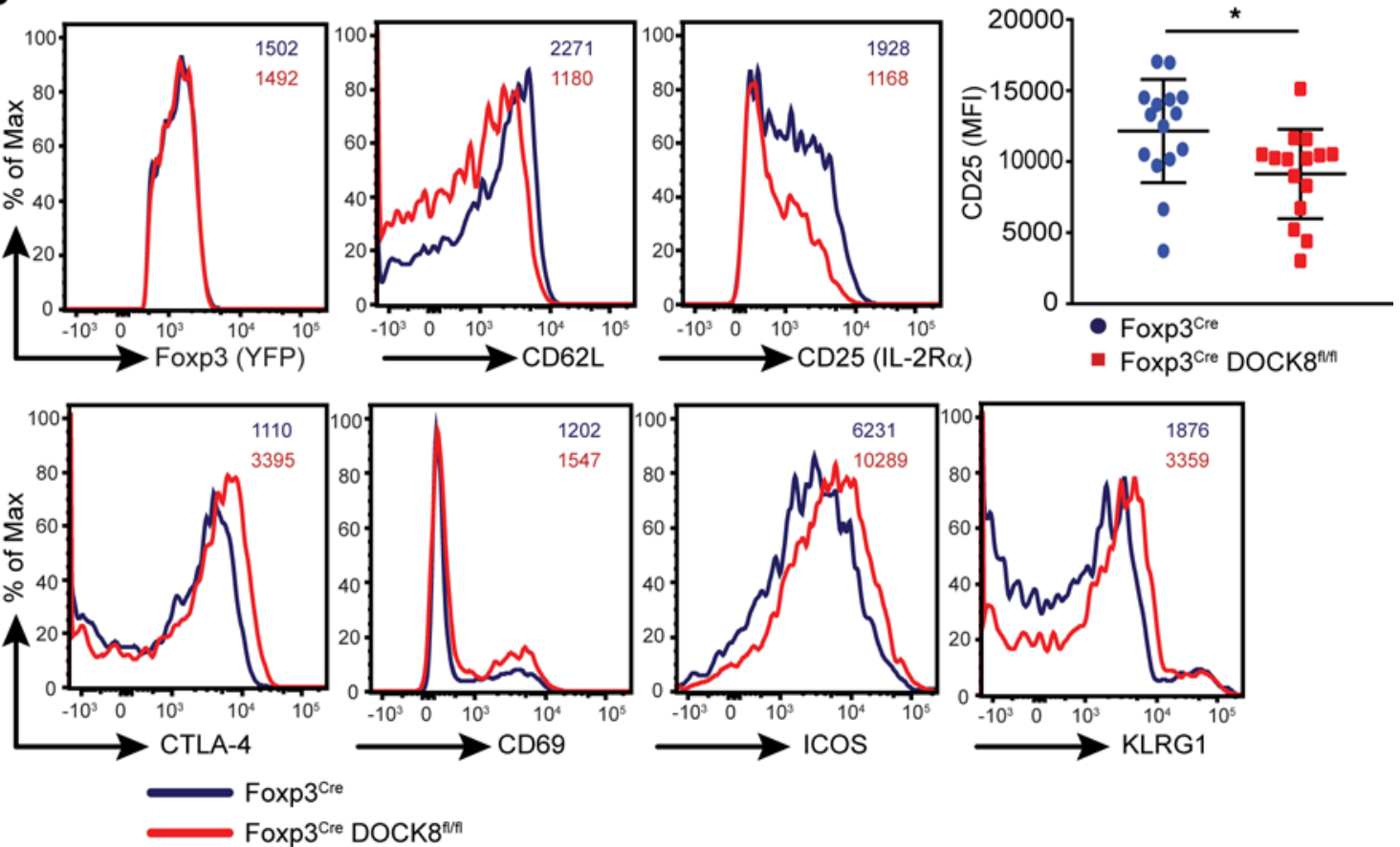

Figure 3. DOCK8 deficiency does not impact the development of Tregs. (A) YFP+ (Foxp3 ${ }^{+}$) Tregs were analyzed in thymus, blood, spleen, LN, and lung by flow cytometry by gating on $\mathrm{CD}_{4} 5^{+} \mathrm{CD} 4^{+} \mathrm{YFP}^{+}$cells. (B) Frequency and absolute numbers of CD45+CD4+ $\mathrm{FFP}^{+}$Tregs from control and Foxp3 ${ }^{\text {Cre }} \mathrm{DOCK} 8^{\mathrm{fl} / \mathrm{fl}}$ mice in indicated organs. Results described in this figure are representative of at least 6 independent experiments with minimum of 2-4 mice per group. (C) Expression of YFP, CD25, CD62L, CTLA-4, ICOS, CD69, and KLRG1 in YFP+ Tregs from control (blue line) or Foxp3 ${ }^{\text {Cre DOCK8 }}{ }^{\text {fl/fl }}$ mice (red line) were determined by flow cytometry analysis and compared. Data represents at least 2 independent experiments with minimum of 3 mice per group. The data shown are the mean \pm SD. Statistics were performed with Prism software by using $t$ test. ${ }^{*} P<0.05$. 
A

A Cont
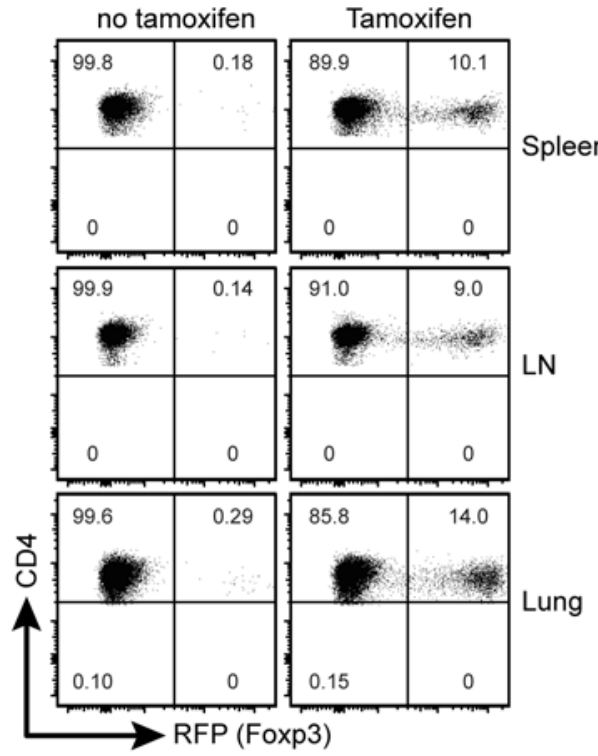

C Cont no tamoxifen Tamoxifen Gated on CD45 ${ }^{+} \mathrm{CD} 4^{+} \mathrm{T}$ cells

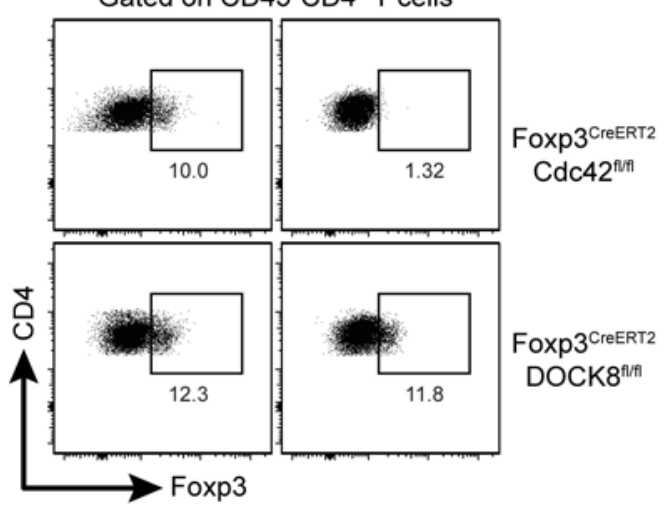

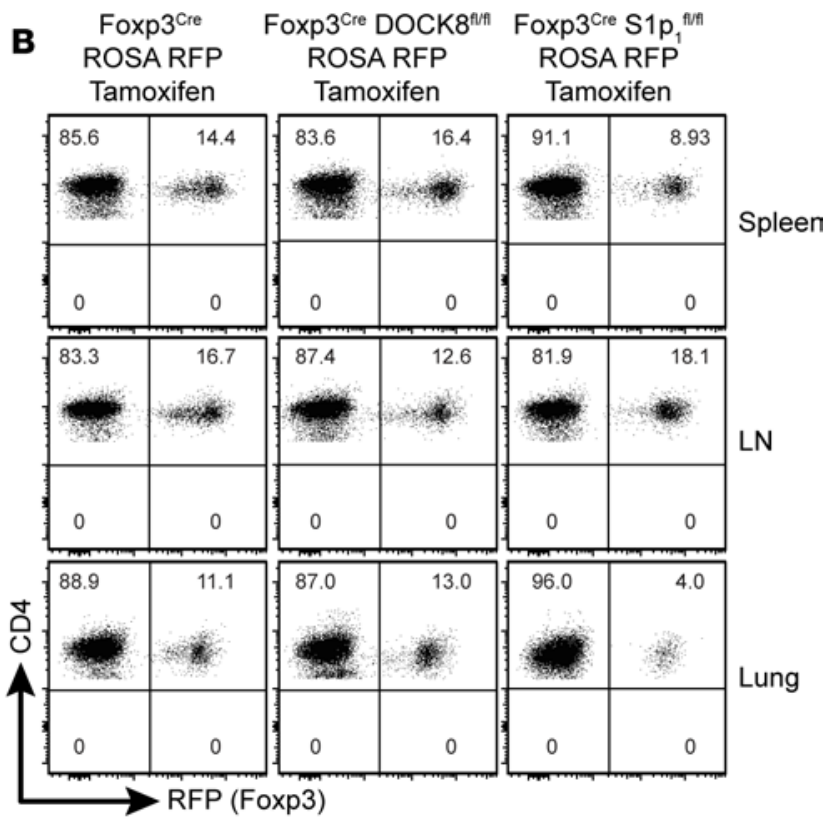

D

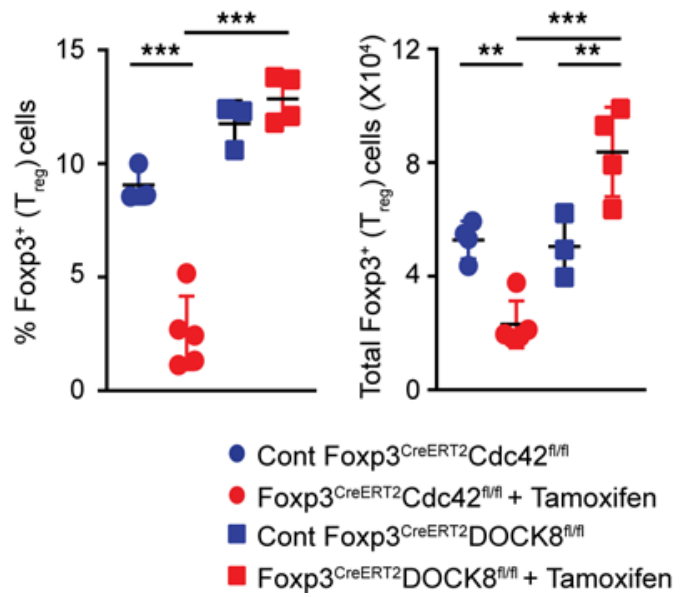

Figure 4. DOCK8 is dispensable for Tregs homing. (A) RFP+ (Foxp3+) Tregs (CD45+CD4+ gated) were analyzed in spleen, LN, and Lungs by flow cytometry at day 2 after tamoxifen injections. Results described in this figure are representative of 2 independent experiments with minimum of 2 mice per group. (B) RFP ${ }^{+}\left(F_{0 x p 3}{ }^{+}\right.$) Tregs (CD45+CD4+ gated) were analyzed in spleen, LNs, and lungs of Foxp3 ${ }^{\text {CreeRT2 }}$ R26RFP, Foxp3 ${ }^{\text {CreERT2}}$ DOCK8 $8^{\text {fl/ff }}$ R26RFP, and Foxp3 ${ }^{\text {CreeRT2 }}$ S1P $_{1}^{\text {fl/fl }}$ R26RFP by flow cytometry at day 10 after tamoxifen injections. Data represents 2 independent experiments with 2-3 mice per group.

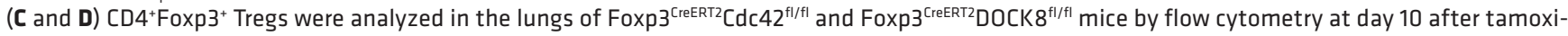
fen injections. FACS plot (C) and scatter plot (D) show the frequency and total Tregs in the lung of control and tamoxifen-injected Foxp3 ${ }^{\text {CreeRT2}}$ DOCK $^{\text {fll }}$ ${ }^{\mathrm{fl}}$ and Foxp $3^{\mathrm{CreERT2}} \mathrm{Cdc} 42^{\mathrm{fl} / \mathrm{fl}}$ mice. Data represents 3 independent experiments with minimum of 3 mice per group. The data shown are the mean $\pm \mathrm{SD}$. Statistics were performed with Prism software by using 1-way ANOVA. ${ }^{* *} P<0.01,{ }^{* *} P<0.001$.

${ }^{2} \mathrm{DOCK} 8^{\mathrm{n} / \mathrm{fl}}$ mice and/or due to defects in their suppressive functions. Further analysis revealed that the frequency of Tregs in the CNS of Foxp $3^{\text {CreeRT2 }}$ DOCK $8^{\mathrm{n} / \mathrm{ll}}$ mice were comparable with those of control Tregs (Figure 6, $\mathrm{D}$ and E). Furthermore, the total number of Tregs in the CNS of Foxp3 ${ }^{\text {CreERT2}} \mathrm{DOCK} 8^{\mathrm{f} / \mathrm{fl}}$ mice was increased due to more lymphocyte infiltration (Figure 6, E-H). These findings suggest that DOCK8-deficient Tregs can migrate to CNS during EAE and populate CNS with similar frequency to those of WT Tregs, but they fail to control CNS inflammation, likely due to defective immune-suppressive function. Thus, we concluded from this experiment that DOCK8 is required for in vivo immune-suppressive function of Tregs.

In order to assess the expression of DOCK8 during the course of EAE, we generated a mouse strain in which flag epitope was knockin in frame with the C-terminal portion of the endogenous DOCK8. The analysis of DOCK8 flag animals revealed that DOCK8 expression was confined to cells of hematopoietic origin 
A
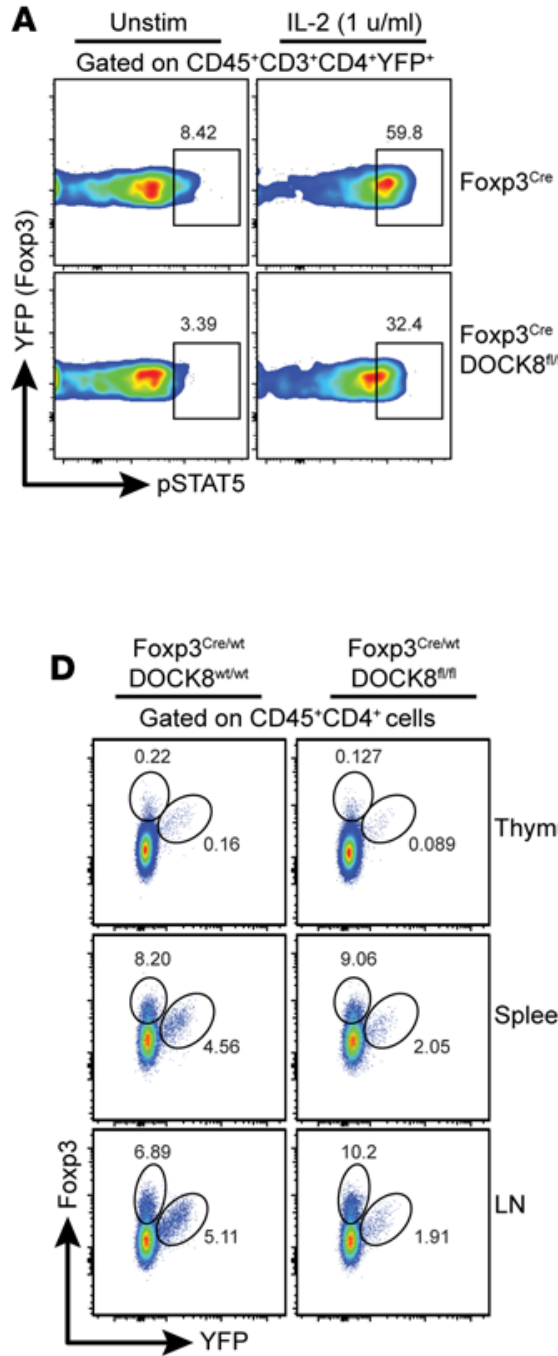

I

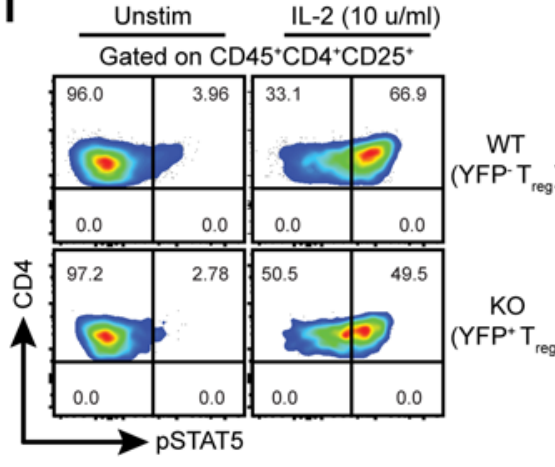

B

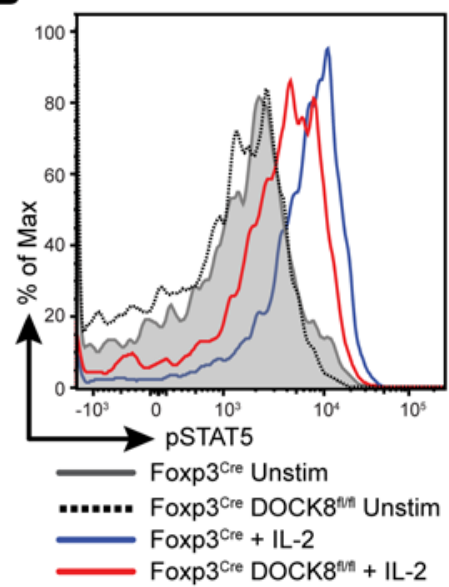

C
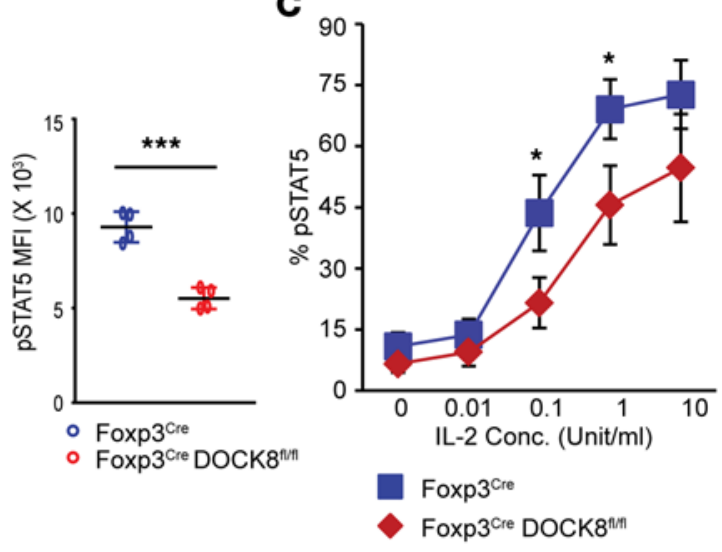

E

$\mathbf{F}$

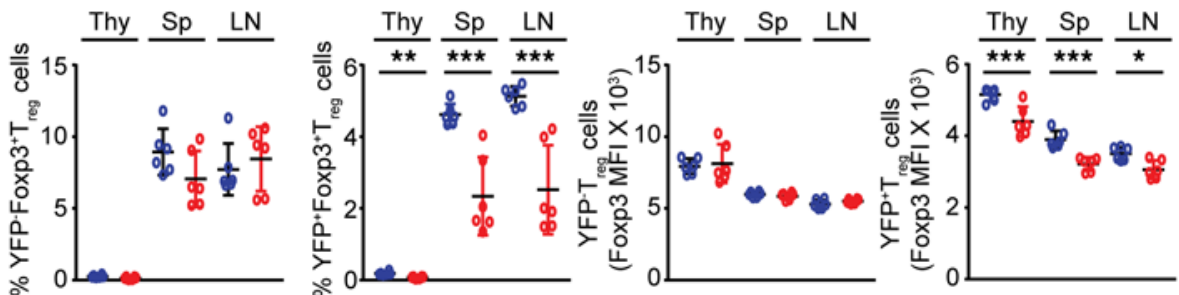

Spleen G Thy $\mathrm{Sp}$ LN
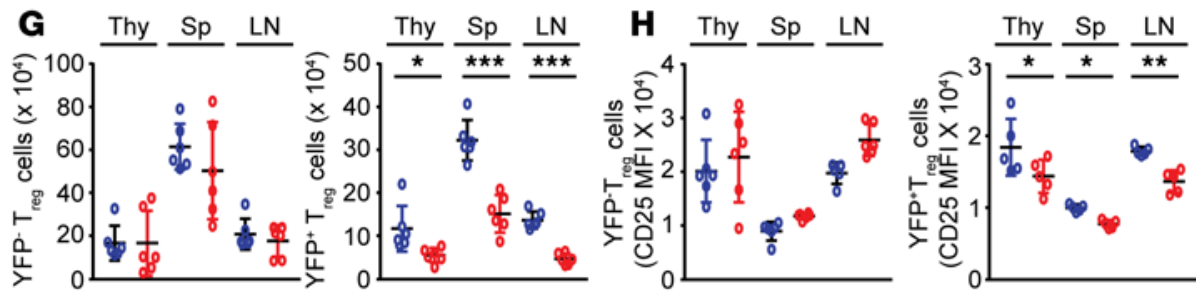

- Foxp3 $3^{\text {crem DOCK }} 8^{\text {mem }} \circ$ Foxp3 $3^{\text {Crem }}$ DOCK8 $8^{\text {mn }}$

J

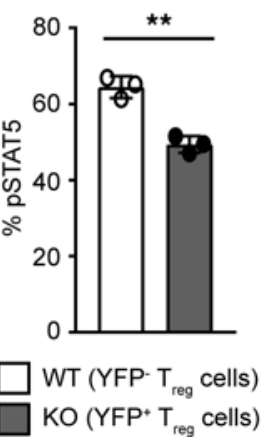

$\mathbf{K}$

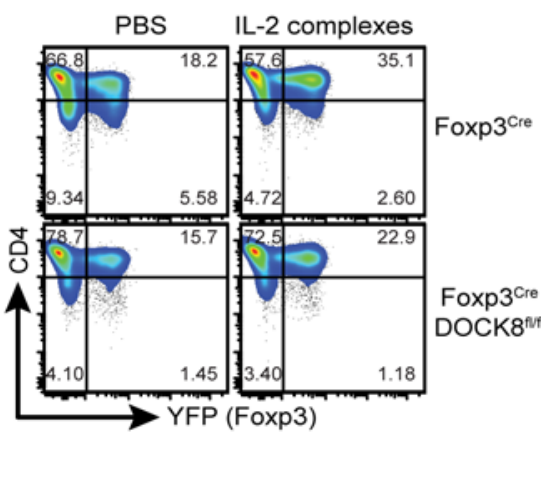

$\mathbf{L}$

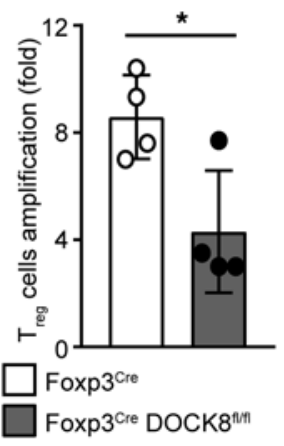

Figure 5. Tregs require DOCK8 for optimal STAT5 activation and competitive fitness. (A-C) Combined cells from spleen and LN were stimulated with indicated concentration of IL-2 for 15 minutes, and then cells were analyzed for pSTAT5 by flow cytometry. (A) FACS plot showing the pSTAT5, (B) histogram showing the mean fluorescent intensity (MFI) of pSTAT5, and (C) dose response analysis of pSTAT5 in response to IL-2 stimulation in CD45 ${ }^{+}$-

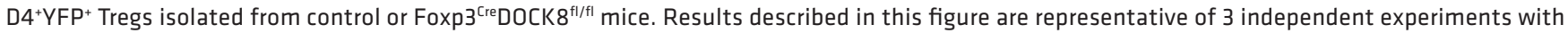
minimum of 2-3 mice per group. (D-H) DOCK8-deficient Tregs are defective in competitive fitness in the presence of WT Tregs. (D) Representative FACS plots showing the frequency of Tregs (CD45+CD4+Foxp3 $\left.3^{+}\right)$in thymus, spleen, and LN of Foxp3 $3^{\text {Cre/wt }}$ DOCK $8^{\text {wt/wt }}$ and Foxp3 $3^{\text {Cre/WT }}$ DOCK $8^{\mathrm{fl} / \mathrm{fl}}$ female mice. (E and $\mathbf{G})$ The frequency (E) and absolute number (C) of Foxp3 ${ }^{+} Y F P^{-}$Tregs (E, left panel) and Foxp $3^{+} Y F P^{+}$Tregs (E, right panel) in indicated organs. (F and $\mathbf{H}$ ) Plot showing the MFI of Foxp3 (F) and CD25 (H) in thymus, spleen, and LN of Foxp3 ${ }^{\mathrm{Cr} / \mathrm{wt}} \mathrm{DOCK} 8^{\mathrm{wt} / \mathrm{wt}}$ and Foxp3 $3^{\mathrm{Cr} / \mathrm{WT}} \mathrm{DOCK} 8^{\mathrm{fl} / \mathrm{fl}}$ female mice. Data represents at least 3 independent experiments with 4-6 mice per group. (I and J) Combined cells from spleen and LN of female Foxp $3^{\text {cre/ }}$ ${ }^{\text {WT }}$ DOCK $8^{\text {fl/fl }}$ mice were stimulated with $10 \mathrm{unit} / \mathrm{ml}$ of IL-2 for 15 minutes, and then cells were analyzed for pSTAT5 by flow cytometry. (I) FACS plot and (J) histogram showing the frequency of pSTAT5 $5^{+}$cells in response to IL-2 stimulation in CD45+CD4+CD25+ YFP- (WT) and CD45+CD4+CD25+YFP+ (KO) Tregs. Results described in these figures are representative of 3 independent experiments with 3 mice per group. (K) Representative FACS plot 


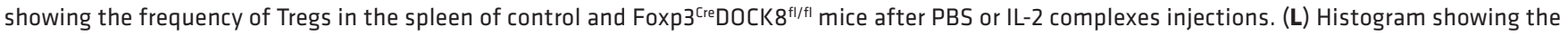

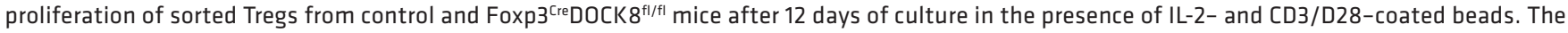
data shown are the mean \pm SD. Statistics were performed with Prism software by using $t$ test. ${ }^{*} P<0.05,{ }^{* *} P<0.01,{ }^{* * *} P<0.001$.

(Figure 6I). Next, we asked whether DOCK8 was expressed during EAE and/or if its expression is altered during the course of disease. Thus, we immunized DOCK8 flag and control animals with $\mathrm{MOG}_{35-55}$ peptide and CFA emulsion, and we analyzed the DOCK8 expression at the peak of disease in spleens and CNS (Figure 6, J and K). As expected, DOCK8 protein was expressed in Foxp3-CD4+ $\mathrm{T}$ cells, Foxp3 ${ }^{+} \mathrm{CD} 4^{+}$Tregs and $\mathrm{CD} 8^{+} \mathrm{T}$ cells in spleens at baseline, and its expression was significantly upregulated at the peak of disease (Figure 6, J, upper panel, and K, left panel). Interestingly, DOCK8 expression was higher in CNS-infiltrating $\mathrm{T}$ cells, with a trend of slightly higher expression in Tregs than effector T cells (Figure 6, J, lower panel, and $\mathrm{K}$, right panel). This data is in line with recent findings (33) in which DOCK8 was shown to upregulate in T cells during EAE and multiple sclerosis (MS). Thus, our results suggest that DOCK8 is crucial for Treg-mediated control of neuroinflammation. It would be interesting to study whether Tregs of MS patients express less DOCK8 than Tregs of healthy individuals. Altogether, our findings indicate that DOCK8 a plays crucial role in immune regulation by controlling Treg biology and their in vivo suppressive function.

\section{Discussion}

In the present study, we have described how specific deficiency of DOCK8 in Tregs leads to compromised immune suppression and homeostasis. Deletion of DOCK8 specifically in Tregs resulted in an autoimmune inflammatory disorder in mice that can be attributed to defective function of Tregs. We have shown that DOCK8 regulates Treg-suppressive function via promoting pSTAT5 in response to IL-2 signaling. Although it is very well established that transcription factor Foxp3 is a master regulator of Tregs, the function of Foxp3 and how Tregs are controlled is not fully understood. In recent years, a number of transcription factors and other molecules have been shown to be important for normal Treg function (34-42). Our studies show that DOCK8 is not required for the development or homing of Tregs, but that it plays an important role in their competitive fitness and immune-suppressive function. Our findings shed new light on the molecular mechanism that regulates the function of Tregs.

A principal finding of our study was that DOCK8 promotes suppressive function of Tregs via positively regulating IL-2 signaling-induced STAT5 phosphorylation. IL-2 signaling-dependent maintenance of Tregs is essential for immune homeostasis $(11,13,14,43,44)$. The suppressive functions of Tregs, in part, can be credited to their ability to effectively compete with effector CD4 $4^{+} \mathrm{T}$ cells for limited IL-2 $(15,45)$. Thus, defective IL-2 signaling on DOCK8-deficient Tregs may lead to defective metabolic fitness, which is essential for maintenance of immune homeostasis and self-tolerance. Our study raises an interesting question of whether DOCK8 directly controls the CD25 transcript or exerts its effect on STAT5. The fact that Foxp $3^{\mathrm{Cre}} \mathrm{DOCK} 8^{\mathrm{n} / \mathrm{fl}}$ mice did not show developmental defects in the thymus and had comparable frequency of Tregs in contrast to IL-2 $2^{-/-}$mice, which have a roughly $50 \%$ reduction in Treg generation (11), and the finding that CD25 transcript levels were comparable between WT and DOCK8-deficient Tregs suggest that DOCK 8 does not directly control CD25 expression. However, reduced cell surface CD25 expression on DOCK8-deficient Tregs might be due to the defective feedback loop required to maintain the IL-2 receptor. In previous studies, the majority of Tregs in IL-2 $2^{--}$mice were either CD25- or CD25 ${ }^{\text {low }}$, which suggested that IL-2 signaling positively regulates the expression of its receptor (11). Based on our results, we would like to propose that DOCK8-deficient Tregs downregulate CD25 expression due to their impaired IL-2-mediated STAT5 phosphorylation. Furthermore, a very recent study has demonstrated that IL-2-mediated activation of STAT5 was critical for the enhancement of Treg-suppressive function to limit ongoing self-stimulating of proto-effector $\mathrm{T}$ cells and maintaining immune tolerance (46). Based on this aforementioned study, compromised STAT5 activation would render suppressive function of Tregs inefficient, which eventually leads to compromised immune tolerance to self. Since Foxp $3^{\mathrm{Cre}} \mathrm{DOCK} 8^{\mathrm{A} / \mathrm{l}}$ mice only showed symptoms after 4 weeks of birth and then expressed a gradually exacerbated autoimmune phenotype, this could suggest that DOCK8-deficient Tregs gradually lost their immune suppressive function due to defective IL-2-mediated signaling. It is well documented that CD25 recycling participates in IL-2-mediated signaling $(47,48)$ and that DOCK8 is also involved in cytoskeletal reorganization $(49,50)$. The defect in IL-2 signaling observed in DOCK8-deficient Tregs could in part be due to abnormal CD25 recycling. However, we failed to rescue DOCK8 deficiency by treating mice with IL-2/anti-IL-2 complexes 
A

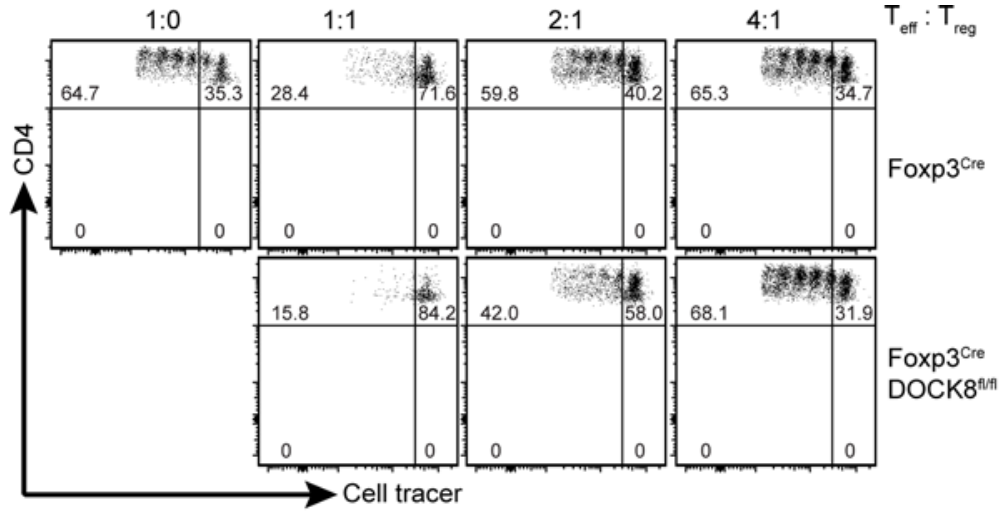

B

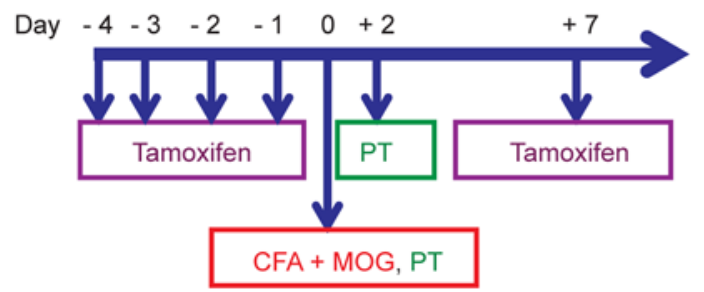

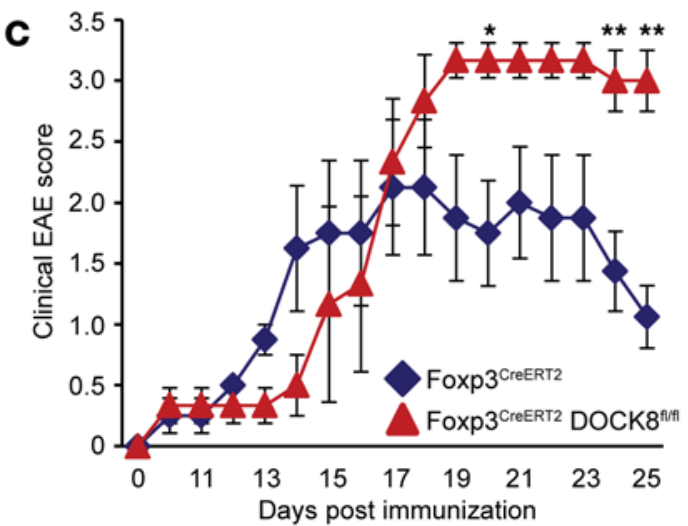
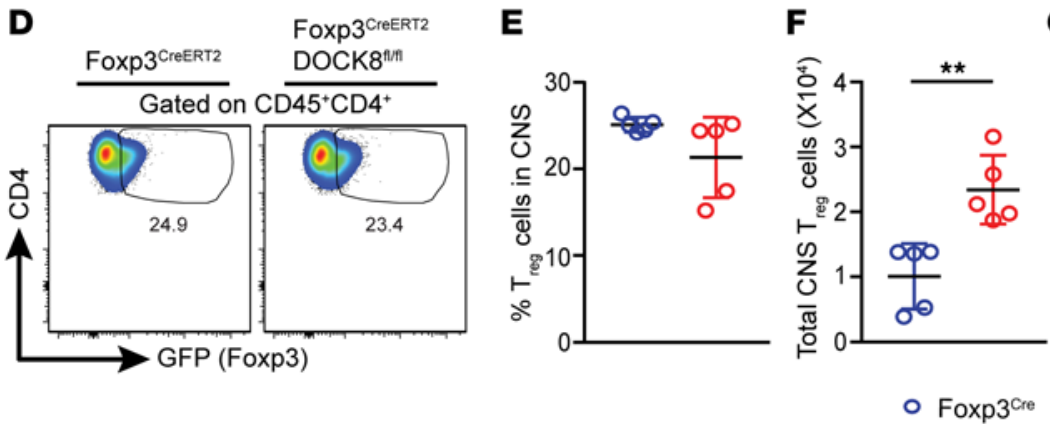

G

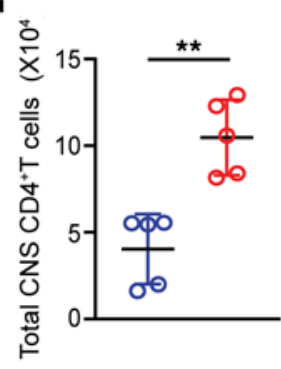

H

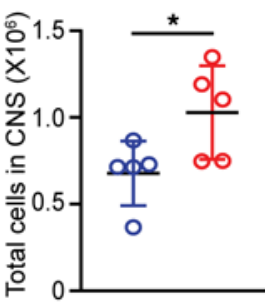

○ Foxp3 $3^{\text {Cre }}$ DOCK8

\section{K}

J
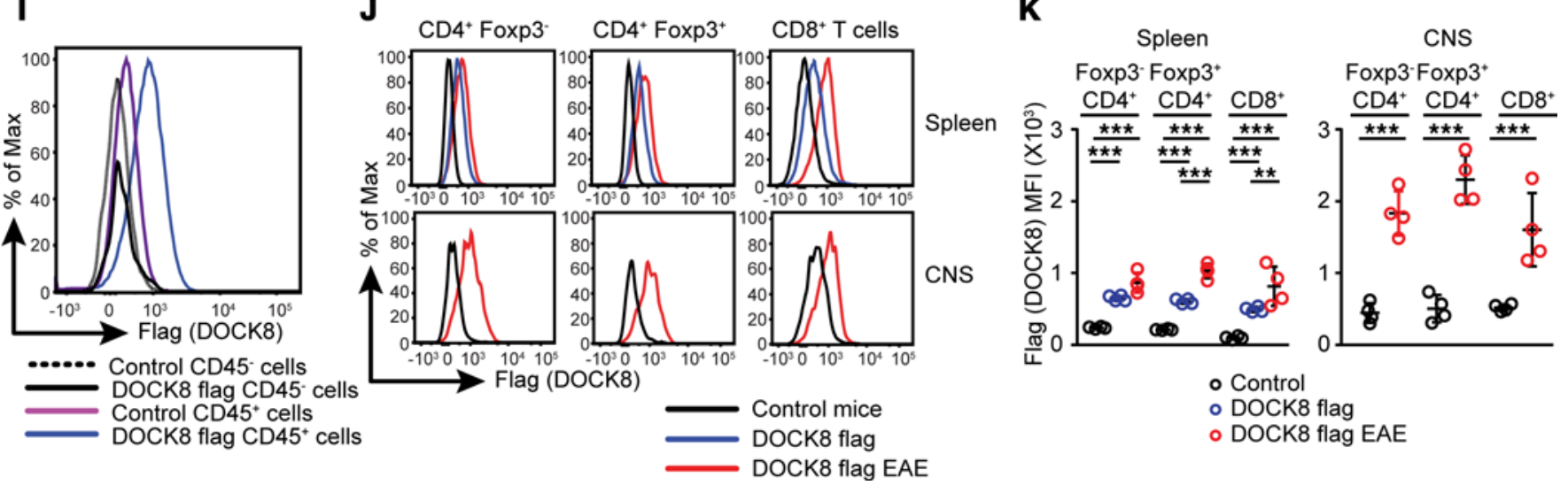

Figure 6. In vivo immune-suppressive functions are impaired in DOCK8-deficient Tregs. (A and B) DOCK8 is dispensable for the in vitro Treg-suppressive function. YFP-CD4+ naive T cells were sorted from control Foxp3 ${ }^{\mathrm{Cre}}$ mice and labeled with cell tracer. In vitro suppression was performed with $5 \times 10^{5}$ irradiated splenocytes as APCs and titrated number of YFP+ Tregs sorted from Foxp $3^{\text {Cre }}$ and Foxp $3^{\text {Cre DOCK }} 8^{\text {fl/fl }}$ mice as suppressor cells. Cultures were stimulated with anti-CD3 $(1 \mu \mathrm{g} / \mathrm{ml})$, and proliferation was measured by loss of cell tracer after 96-hour stimulation. Data are representative of 2 independent experiments. (B-H) DOCK8-deficient Tregs failed to suppress in vivo inflammation during EAE a mouse model to study CNS inflammation. (B) Schematic representation showing the DOCK8 deletion by tamoxifen injection and induction of EAE in 8- to 10-week-old control Foxp3 ${ }^{\text {CreERT2 }}$ mice and Foxp $3^{\text {CreeRT2}}$ DOCK $8^{\text {fl/ }}$ 
fl mice. (C) Control (Foxp3 ${ }^{\text {CreeRT2}}$ ) and Foxp3 $3^{\text {CreeRT2}}{ }^{\text {DOCK }} 8^{\text {fl/fl }}$ mice were immunized with MOC $^{35-55}$ in CFA and monitored for clinical signs of EAE. (D and E) Frequency and (F) absolute number of Tregs in the CNS of control and Foxp3 ${ }^{\text {CreeRT2}}$ DOCK $8^{\mathrm{fl} / \mathrm{fl}}$ mice at the peak of disease. (C) The total CNS-infiltrating CD4 ${ }^{+}$ T cells and $(\mathbf{H})$ absolute number of total CNS infiltrating lymphocytes. Data are representative of 3 separate experiments with 5 mice per group. The data shown are the mean \pm SD. Statistics were performed either with Prism software by using $t$ test (2-tailed) (in C, E-H) and Mann-Whitney $U$ test (in $\mathbf{C}$ ). ${ }^{*} P$ $<0.05$, ${ }^{*} P<0.01$. (I-K) DOCK8 flag tag mice showing the expression of DOCK8 on hematopoietic cells including Tregs, and its expression is upregulated during CNS inflammation. (I) Flow histogram showing that DOCK8 is specifically expressed on hematopoietic cells. (J and K) Control or DOCK8 flag tag mice were immunized with MOG $\mathrm{C}^{35-55}$ in CFA, and their spleen and CNS were analyzed for DOCK8 expression at the peak of disease. (J) Upper panel shows DOCK8 expression in spleen at baseline (blue line) and at the peak of EAE (red line). Lower panel shows the DOCK8 expression at the peak of EAE (red line). (K) The MFI of DOCK8 on Foxp3-CD4 ${ }^{+} T$ cells, Foxp3 ${ }^{+} \mathrm{CD} 4^{+} \mathrm{T}$ cells, and $\mathrm{CD} 8^{+} \mathrm{T}$ cells in the spleen and CNS. Data represents 2-3 independent experiments with 3-6 mice per group. The data shown are the mean \pm SD. Statistics were performed with Prism software by using 1 -way ANOVA. ${ }^{* *} P<0.01,{ }^{* *} P<0.001$.

that should have overcome the need for CD25 recycling. Thus, how DOCK8 regulates STAT5 activation in response to IL-2 will require further study. Our in vivo data indicated that the function of DOCK8-deficient Tregs is compromised because acute deletion of DOCK8 in Tregs during EAE renders mice susceptible to disease, despite having comparable numbers of Tregs in the CNS. However, in vitro, DOCK8 expression was not required for the suppressive activity of Tregs. It is well documented that in vitro Treg-suppressive assays may not necessarily mimic the in vivo Treg-suppressive function (51).

DOCK8-deficient patients develop Staphylococcus aureus skin abscesses, or soft tissue infections, elevated serum IgE, eosinophilia, and GI tract infections (19). A recent study has also found that memory CD4 ${ }^{+}$ T cells of DOCK8-deficient patients are skewed toward Th2 cells, probably at the expense of Th1 and Th17 cells (52), which may result in a compromised antiviral and antifungal immunity.

DOCK8-deficient patients are also susceptible to autoimmunity and develop colitis, autoimmune hemolytic anemia, and vasculitis (53-55). These patients display increased levels of autoantibodies due to defective peripheral B cell tolerance, which was correlated with reduced numbers of Tregs with impaired suppressive function (28). Thus, autoimmune phenotypes in DOCK8-deficient patients may indicate a possible loss of immune tolerance. However, these patients have combined immunodeficiency that makes it complicated to identify the specific defect in a particular cell. For example, DOCK8 deficiency causes $\mathrm{T}$ cell lymphopenia and functional defect in these cells, making it harder to distinguish if DOCK8 is also specifically required in Tregs. In addition, phenotypes observed in DOCK8-deficient patients could be due to secondary causes or loss of Treg-suppressive function due to persistent ongoing inflammation. Therefore, it was crucial to understand whether DOCK8 also contributed to immune tolerance via directly controlling Tregs. Our study therefore suggests that DOCK 8 is required for immune tolerance via directly controlling IL-2 signaling in Tregs and is, thus, essential for maintaining immune homeostasis.

In summary, our data shows that the intrinsic requirement of DOCK8 in Tregs is crucial for immune tolerance. Finally, our study provides potentially novel insights into the essential function of DOCK8 in Tregs and immune regulation, and it may explain the autoimmune manifestations associated with DOCK8 deficiency.

\section{Methods}

Mice. Dock $8^{f / f l}$ mice were generated by introducing the loxp site upstream of exon 42 and downstream of exon 43 and bred in house to Foxp3 $3^{\text {CreYFP }}$, which were a gift from A. Rudensky (Memorial Sloan Kettering Cancer Center, New York, NY, USA), at specific pathogen-free conditions. Foxp3 ${ }^{\text {CreERT2 }}$ mice were purchased from Jackson Laboratories (STOCK Foxp3tm9[EGFP/cre/ERT2]Ayr/J) and bred to Dock8fl/fl on C57BL/6 background. Dock8 flag tag mouse was generated by in-frame knockin of flag epitope with the C-terminal portion of endogenous Dock8. Dock8 ${ }^{\text {pri/pri }}(25), \mathrm{Cdc}^{\mathrm{ft} / \mathrm{fl}}(56)$, and $\mathrm{S}^{\mathrm{P}} \mathrm{P}_{1}^{\mathrm{fl} / \mathrm{fl}}(57)$ mice were generated as previously described. All the mice were bred and maintained under specific pathogen-free conditions.

Flow cytometric analysis and cell sorting. Cells were isolated from various organs as described previously (58). Most of the mice used in this study were 8-10 weeks old. Both male and female mice were used in this study, but their sexes were matched accordingly with test group. Fluorescence-conjugated antibodies for CD45 (clone 30-F11), CD4 (clone L3T4), CD8 (clone 53-6.7), CD25 (clone PC61.5), CD44 (clone IM7), CD69 (clone H1.2F3), CTLA-4 (clone UC10-4B9), Ki-67 (clone SolA15), pSTAT5 (clone SRBCZX), and anti-Flag (clone FG4R) (all purchased from eBioscience), as well as Foxp3 (clone 150D), IFN- $\gamma$ (clone XMG1.2), IL-17A (clone eBio 17B7), CD62L (clone MEL-14), ICOS (clone C398.4A), KLRG-1 (clone 2F1/KLRG1) (purchased from BioLegend) were used. Cell surface and intracellular staining was performed according to manufacturer's protocols. For cytokine production, cells were stimulated directly ex vivo by incubating for 4 hours with $50 \mathrm{ng} / \mathrm{ml}$ PMA (MilliporeSigma) and $1 \mu \mathrm{g} / \mathrm{ml}$ Ionomycine (Milli- 
poreSigma) in the presence of Golgi plug (BD Biosciences). Cell were fixed and permeabilized by using cytofix/cytoperm solution (catalog 554722, BD Biosciences), as described by manufacturers, and stained with IL-17A and IFN- $\gamma$. For Foxp3 staining, cells were fixed and permeabilized by using Foxp3 fix/perm buffer set (catalog 421403, BioLegend), as described by manufacturers, and stained with Foxp3.

In vitro Treg suppression assay. Tregs were sorted from 8- to 10-week-old controls or Foxp3 ${ }^{\mathrm{Cre}} \mathrm{DOCK} 88^{\mathrm{f} / \mathrm{fl}}$ mice (LN and spleen combined) after initial positive enrichment step via CD4 $4^{+}$microbeads (Miltenyi Biotec). Naive $\mathrm{CD}^{+} \mathrm{T}$ cells were sorted only from control mice and then labeled with cell tracer before their addition in suppression assay. Tregs were sorted based on $\mathrm{CD} 45^{+} \mathrm{CD} 4^{+} \mathrm{YFP}^{+}$and $\mathrm{CD} 25^{+}$. For in vitro Treg suppression assay, we used $5 \times 10^{5}$ irradiated antigen presenting cells (APCs) together with $1 \times 10^{5}$ naive $\mathrm{CD} 4^{+} \mathrm{T}$ cells and titrated the number of Tregs from control and Foxp $3^{\mathrm{Cre}} \mathrm{DOCK} 8^{\mathrm{f} / \mathrm{fl}}$ mice in the presence of $1 \mu \mathrm{g} / \mathrm{ml}$ anti-CD3 (eBioscience).

Determination of pSTAT5 levels. To examine IL-2 signaling, $1 \times 10^{6}$ lymphocytes from spleen of control

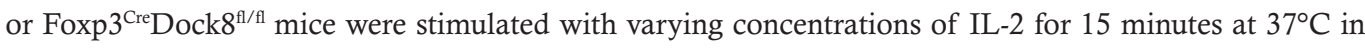
a 96-well plate (100 $\mu$ volume). Cells were fixed with the addition of equal volume of $4 \%$ formalin for 10 minutes. Cells were permeabilized with Perm Buffer III (BD Biosciences), and staining of pSTAT5 (pY694) was performed according to manufacturer's protocol.

$I L-2$ and IL-2 mAb complex injections. Foxp $3^{\mathrm{Cre}}$ and Foxp $3^{\mathrm{Cre}} \mathrm{DOCK} 8^{\mathrm{f} / \mathrm{fl}}$ mice were given i.p. injections of IL-2 complex at day 0,1 , and 2, and animals were analyzed at day 5 for Treg proliferation. IL-2 complexes were prepared by mixing $1 \mu \mathrm{g}$ mouse IL-2 (eBioscience) with $5 \mu \mathrm{g}$ mouse anti-IL-2 mAb (clone JES6$1 \mathrm{~A} 12$, eBioscience), followed by 30 minutes of incubation at $37^{\circ} \mathrm{C}$.

Induction and assessment of EAE. Foxp $3^{\mathrm{CreERT} 2}$ and Foxp3 $3^{\mathrm{CreERT} 2} \mathrm{DOCK} 8^{\mathrm{fl} / \mathrm{fl}}$ mice were injected s.c. with 100 $\mu \mathrm{g}$ of $\mathrm{MOG}_{35-55}$ peptide (MEVGWYRSPFSRVVHLYRNGK) emulsified in CFA (Difco) supplemented with 4 $\mathrm{mg} / \mathrm{ml}$ Mycobacterium tuberculosis extract H37Ra (Difco). Additionally, mice received $200 \mathrm{ng}$ pertussis toxin (List Biological Laboratories) i.p. at day 0 and 2. Clinical assessment of EAE was performed daily after disease induction according to the following conditions: no disease, 0 ; reduced tail tone, 1 ; hindlimb weakness, 2 ; hindlimb paralysis, 3; forelimb and hindlimb paralysis, 4; and moribund, 5.

CNS cell isolation. CNS mononuclear cells were isolated at the peak of disease. Mice were euthanized by using $\mathrm{CO}_{2}$, followed by perfusion through the left cardiac ventricle with PBS to remove circulating lymphocytes. In order to harvest CNS, subcranial structures were dissected from cerebrum, while spinal cord was flushed out with PBS. Both CNS and spinal cord tissues were combined and cut into small pieces, followed by enzymatic digestion in the presence of $1.5-\mathrm{mg} / \mathrm{ml}$ collagenase type IV and $0.25 \mathrm{mg} / \mathrm{ml} \mathrm{DNaseI}$ at $37^{\circ} \mathrm{C}$ in an incubator shaker for 45 minutes. The digested tissues were then filtered through a $40-\mu \mathrm{m}$ filter and centrifuged. The cell pellet was resuspended in $5 \mathrm{ml}$ of the $70 \%$ fraction of a 70:37 Percoll gradient and overlaid on $5 \mathrm{ml}$ of the $37 \%$ fraction in a $15 \mathrm{ml}$ Falcon tube (Genesee Scientific). Percoll gradient separation was performed by centrifugation without break for 20 minutes at $788 \mathrm{~g}$. Mononuclear cells were removed from the interphase, washed with cold PBS, and resuspended in culture medium for analysis.

Tamoxifen treatments. Tamoxifen (MilliporeSigma) was dissolved in corn oil (MilliporeSigma) to a solution of $15 \mathrm{mg} / \mathrm{ml}$ by shaking at $37^{\circ} \mathrm{C}$ and kept at $4^{\circ} \mathrm{C}$ until use. Mice were injected i.p. with $100 \mu 1$ from 15 $\mathrm{mg} / \mathrm{ml}$ stock (total $1.5 \mathrm{mg}$ for mice weighing $20 \mathrm{~g}$ ). Tamoxifen injections were repeated for 4 consecutive days, and mice were sacrificed at indicated times. For EAE experiments, mice were injected for 4 consecutive days, immunized with MOG, and then injected at day 7 after immunization.

Measurement of autoantibodies. Autoantibody titers were measured by ELISA as previously described (59). Briefly, 96-well Immuno plates (Nunc) were precoated overnight at $4^{\circ} \mathrm{C}$ with calf thymus dsDNA (100 $\mu \mathrm{g} / \mathrm{ml}$; MilliporeSigma, D3664-5X2MG) or Sm/RNP (5 $\mathrm{g} / \mathrm{ml}$; Arotec Diagnostic Limited, ATR01-10). After blocking in $1 \%$ BSA, plates were incubated with diluted serum, and specific antibody isotypes were detected using goat anti-mouse IgM- (catalog 1020-02), IgG- (catalog 1030-50), or IgG2c-HRP (catalog 1079-05) (1:2,000 dilution; Southern Biotechnology Associates), followed by measurement of peroxidase reactions using OptEIA TMB substrate (BD Biosciences).

Histology. Lungs were fixed in 10\% formalin overnight and, the next day, were transferred in to $70 \%$ ethanol. Paraffin embedded blocks were sectioned (4-5 $\mu \mathrm{m})$ and H\&E stained.

Statistics. For statistical analysis, $P$ values were calculated by using either Student's $t$ test, 1 -way ANOVA, or Mann-Whitney U test. $P$ values of less than 0.05 were considered significant. Error bars denote \pm SD.

Study approval. All the experiments in animals were reviewed and approved by the IACUC of Seattle Children's Research Institute and were performed accordance with the institutional guidelines. 


\section{Author contributions}

AKS designed, performed, and analyzed most experiments and wrote the manuscript. AE, KK, GB, and AS characterized Treg populations. DH helped with pSTAT5 assays. TA and SWJ performed autoantibodies using ELISA. TRT and EB designed and analyzed the experiments. MO supervised the study, analyzed the data, and edited the manuscript.

\section{Acknowledgments}

We thank Christopher Goodnow (Australian National University) for providing us the Dock8 ${ }^{\text {pripri }}$ mice, Roy Zent (University of Vanderbilt) for providing the Cdc42 $2^{\mathrm{fl} / \mathrm{fl}}$, and Richard L. Proia (NIH) for his gift of $\mathrm{S}_{1} \mathrm{P}_{1}^{\mathrm{f} / \mathrm{fl}}$ mice. We would like to thank Brian Johnson from Histology and Imaging Core Facility at University of Washington, Seattle for his help with histology; Maia Chan, Devin Margolies, and Stephanie Slater for assistance with animal husbandry; and Gene Hess from Flow Cytometry and Cell sorting core facility at SCRI. This work was funded by NIH grants 1R21AI124735-0 and R56 AI113906 and National Multiple Sclerosis society grant RG-5044-A-2 to MO. We would also like to thank Dawn Marie Pares for her outstanding administrative assistance.

Address correspondence to: Mohamed Oukka, Seattle Research Institute, 1900 Ninth Avenue, Seattle, Washington 98101, USA. Phone: 206.884.1140; Email: moukka@u.washington.edu.

1. Hori S, Nomura T, Sakaguchi S. Control of regulatory T cell development by the transcription factor Foxp3. Science. 2003;299(5609):1057-1061.

2. Fontenot JD, Gavin MA, Rudensky AY. Foxp3 programs the development and function of $\mathrm{CD}^{+} \mathrm{CD} 25^{+}$regulatory T cells. $\mathrm{Nat}$ Immunol. 2003;4(4):330-336.

3. Khattri R, Cox T, Yasayko SA, Ramsdell F. An essential role for Scurfin in CD $4^{+} \mathrm{CD} 25^{+}$T regulatory cells. Nat Immunol. 2003;4(4):337-342.

4. Sakaguchi S, Yamaguchi T, Nomura T, Ono M. Regulatory T cells and immune tolerance. Cell. 2008;133(5):775-787.

5. Shevach EM. Regulatory T cells in autoimmmunity*. Annu Rev Immunol. 2000;18:423-449.

6. Liston A, Rudensky AY. Thymic development and peripheral homeostasis of regulatory T cells. Curr Opin Immunol. 2007;19(2):176-185.

7. Brunkow ME, et al. Disruption of a new forkhead/winged-helix protein, scurfin, results in the fatal lymphoproliferative disorder of the scurfy mouse. Nat Genet. 2001;27(1):68-73.

8. Wildin RS, et al. X-linked neonatal diabetes mellitus, enteropathy and endocrinopathy syndrome is the human equivalent of mouse scurfy. Nat Genet. 2001;27(1):18-20.

9. Bennett CL, et al. The immune dysregulation, polyendocrinopathy, enteropathy, X-linked syndrome (IPEX) is caused by mutations of FOXP3. Nat Genet. 2001;27(1):20-21.

10. Josefowicz SZ, Lu LF, Rudensky AY. Regulatory T cells: mechanisms of differentiation and function. Annu Rev Immunol. 2012;30:531-564.

11. Fontenot JD, Rasmussen JP, Gavin MA, Rudensky AY. A function for interleukin 2 in Foxp3-expressing regulatory T cells. Nat Immunol. 2005;6(11):1142-1151.

12. Setoguchi R, Hori S, Takahashi T, Sakaguchi S. Homeostatic maintenance of natural Foxp3(+) CD25(+) CD4(+) regulatory T cells by interleukin (IL)-2 and induction of autoimmune disease by IL-2 neutralization. J Exp Med. 2005;201(5):723-735.

13. Antov A, Yang L, Vig M, Baltimore D, Van Parijs L. Essential role for STAT5 signaling in CD $25^{+} \mathrm{CD} 4^{+}$regulatory T cell homeostasis and the maintenance of self-tolerance. J Immunol. 2003;171(7):3435-3441.

14. Cheng G, Yu A, Malek TR. T-cell tolerance and the multi-functional role of IL-2R signaling in T-regulatory cells. Immunol Rev. 2011;241(1):63-76.

15. Burchill MA, Yang J, Vogtenhuber C, Blazar BR, Farrar MA. IL-2 receptor $\beta$-dependent STAT5 activation is required for the development of Foxp3 ${ }^{+}$regulatory T cells. J Immunol. 2007;178(1):280-290.

16. Yao Z, et al. Nonredundant roles for Stat5a/b in directly regulating Foxp3. Blood. 2007;109(10):4368-4375.

17. Randall KL, Lambe T, Goodnow CC, Cornall RJ. The essential role of DOCK 8 in humoral immunity. Dis Markers. 2010;29(3-4):141-150.

18. Su HC. Dedicator of cytokinesis 8 (DOCK8) deficiency. Curr Opin Allergy Clin Immunol. 2010;10(6):515-520.

19. Su HC, Jing H, Zhang Q. DOCK8 deficiency. Ann N Y Acad Sci. 2011;1246:26-33.

20. Crawford G, et al. DOCK8 is critical for the survival and function of NKT cells. Blood. 2013;122(12):2052-2061.

21. Harada Y, et al. DOCK8 is a Cdc42 activator critical for interstitial dendritic cell migration during immune responses. Blood. 2012;119(19):4451-4461.

22. Jabara $\mathrm{HH}$, et al. DOCK8 functions as an adaptor that links TLR-MyD88 signaling to B cell activation. Nat Immunol. $2012 ; 13(6): 612-620$.

23. Lambe T, et al. DOCK8 is essential for T-cell survival and the maintenance of CD8 $8^{+} \mathrm{T}$-cell memory. Eur J Immunol. 2011;41(12):3423-3435

24. Randall KL, et al. DOCK8 deficiency impairs CD8 T cell survival and function in humans and mice. J Exp Med. 2011;208(11):2305-2320. 
25. Randall KL, et al. Dock8 mutations cripple B cell immunological synapses, germinal centers and long-lived antibody production. Nat Immunol. 2009;10(12):1283-1291

26. Zhang Q, et al. DOCK8 regulates lymphocyte shape integrity for skin antiviral immunity. J Exp Med. 2014;211(13):2549-2566.

27. Singh AK, Eken A, Fry M, Bettelli E, Oukka M. DOCK8 regulates protective immunity by controlling the function and survival of ROR $\gamma \mathrm{t}^{+}$ILCs. Nat Commun. 2014;5:4603.

28. Janssen E, et al. Dedicator of cytokinesis 8-deficient patients have a breakdown in peripheral B-cell tolerance and defective regulatory T cells. J Allergy Clin Immunol. 2014;134(6):1365-1374.

29. Rubtsov YP, et al. Regulatory T cell-derived interleukin-10 limits inflammation at environmental interfaces. Immunity. 2008;28(4):546-558.

30. Matloubian M, et al. Lymphocyte egress from thymus and peripheral lymphoid organs is dependent on S1P receptor 1. Nature 2004;427(6972):355-360.

31. Guo F, Hildeman D, Tripathi P, Velu CS, Grimes HL, Zheng Y. Coordination of IL-7 receptor and T-cell receptor signaling by cell-division cycle 42 in T-cell homeostasis. Proc Natl Acad Sci U S A. 2010;107(43):18505-18510.

32. McGeachy MJ, Stephens LA, Anderton SM. Natural recovery and protection from autoimmune encephalomyelitis: contribution of $\mathrm{CD}^{+} \mathrm{CD} 25^{+}$regulatory cells within the central nervous system. J Immunol. 2005;175(5):3025-3032.

33. Xu X, et al. LRCH1 interferes with DOCK8-Cdc42-induced T cell migration and ameliorates experimental autoimmune encephalomyelitis. J Exp Med. 2017;214(1):209-226.

34. Chaudhry A, et al. CD4 ${ }^{+}$regulatory T cells control TH17 responses in a Stat3-dependent manner. Science. 2009;326(5955):986-991

35. Kerdiles YM, et al. Foxo transcription factors control regulatory T cell development and function. Immunity. 2010;33(6):890-904.

36. Kitoh A, et al. Indispensable role of the Runx1-Cbf $\beta$ transcription complex for in vivo-suppressive function of FoxP $3^{+}$regulatory T cells. Immunity. 2009;31(4):609-620.

37. Koch MA, Tucker-Heard G, Perdue NR, Killebrew JR, Urdahl KB, Campbell DJ. The transcription factor T-bet controls regulatory T cell homeostasis and function during type 1 inflammation. Nat Immunol. 2009;10(6):595-602.

38. Ouyang W, Beckett O, Ma Q, Paik JH, DePinho RA, Li MO. Foxo proteins cooperatively control the differentiation of Foxp3 regulatory T cells. Nat Immunol. 2010;11(7):618-627.

39. Ouyang W, et al. Novel Foxo1-dependent transcriptional programs control T(reg) cell function. Nature. 2012;491(7425):554-559.

40. Rudra D, Egawa T, Chong MM, Treuting P, Littman DR, Rudensky AY. Runx-CBF $\beta$ complexes control expression of the transcription factor Foxp3 in regulatory T cells. Nat Immunol. 2009;10(11):1170-1177.

41. Wang Y, Su MA, Wan YY. An essential role of the transcription factor GATA-3 for the function of regulatory T cells. Immunity 2011;35(3):337-348.

42. Zheng Y, et al. Regulatory T-cell suppressor program co-opts transcription factor IRF4 to control T(H)2 responses. Nature. 2009;458(7236):351-356.

43. Thornton AM, Donovan EE, Piccirillo CA, Shevach EM. Cutting edge: IL-2 is critically required for the in vitro activation of $\mathrm{CD}^{+} \mathrm{CD} 25^{+} \mathrm{T}$ cell suppressor function. J Immunol. 2004;172(11):6519-6523.

44. Sakaguchi S, Wing K, Onishi Y, Prieto-Martin P, Yamaguchi T. Regulatory T cells: how do they suppress immune responses? Int Immunol. 2009;21(10):1105-1111.

45. Pandiyan P, Zheng L, Ishihara S, Reed J, Lenardo MJ. CD4 ${ }^{+} \mathrm{CD} 25^{+}$Foxp $3^{+}$regulatory T cells induce cytokine deprivation-mediated apoptosis of effector CD4+ T cells. Nat Immunol. 2007;8(12):1353-1362.

46. Liu Z, Gerner MY, Van Panhuys N, Levine AG, Rudensky AY, Germain RN. Immune homeostasis enforced by co-localized effector and regulatory T cells. Nature. 2015;528(7581):225-230.

47. Su EW, et al. IL-2R $\alpha$ mediates temporal regulation of IL-2 signaling and enhances immunotherapy. Sci Transl Med. 2015;7(311):311ra170.

48. Ellery JM, Nicholls PJ. Possible mechanism for the alpha subunit of the interleukin-2 receptor (CD25) to influence interleukin-2 receptor signal transduction. Immunol Cell Biol. 2002;80(4):351-357.

49. McGhee SA, Chatila TA. DOCK8 immune deficiency as a model for primary cytoskeletal dysfunction. Dis Markers. 2010;29(34):151-156

50. Martins R, et al. Heme drives hemolysis-induced susceptibility to infection via disruption of phagocyte functions. Nat Immunol. 2016;17(12):1361-1372.

51. Mercadante ER, Lorenz UM. Breaking free of control: how conventional T cells overcome regulatory T cell suppression. Front Immunol. 2016;7:193.

52. Tangye SG, et al. Dedicator of cytokinesis 8-deficient CD4(+) T cells are biased to a TH2 effector fate at the expense of TH1 and TH17 cells. J Allergy Clin Immunol. 2017;139(3):933-949.

53. Engelhardt KR, et al. Large deletions and point mutations involving the dedicator of cytokinesis 8 (DOCK8) in the autosomal-recessive form of hyper-IgE syndrome. J Allergy Clin Immunol. 2009;124(6):1289-302.e4.

54. Sanal O, et al. Additional diverse findings expand the clinical presentation of DOCK8 deficiency. J Clin Immunol. 2012;32(4):698-708.

55. Zhang Q, et al. Combined immunodeficiency associated with DOCK8 mutations. N Engl J Med. 2009;361(21):2046-2055.

56. Wu X, et al. Cdc42 controls progenitor cell differentiation and beta-catenin turnover in skin. Genes Dev. 2006;20(5):571-585.

57. Allende ML, Yamashita T, Proia RL. G-protein-coupled receptor S1P1 acts within endothelial cells to regulate vascular maturation. Blood. 2003;102(10):3665-3667.

58. Sather BD, et al. Altering the distribution of Foxp3(+) regulatory T cells results in tissue-specific inflammatory disease. $J$ Exp Med. 2007;204(6):1335-1347.

59. Jackson SW, et al. B cell IFN- $\gamma$ receptor signaling promotes autoimmune germinal centers via cell-intrinsic induction of BCL-6. JExp Med. 2016;213(5):733-750. 